\title{
Amplitude Expansions for Instabilities in Populations of Globally-Coupled Oscillators
}

\author{
John David Crawford \\ Department of Physics and Astronomy \\ University of Pittsburgh \\ Pittsburgh, PA 15260
}

\begin{abstract}
We analyze the nonlinear dynamics near the incoherent state in a mean-field model of coupled oscillators. The population is described by a Fokker-Planck equation for the distribution of phases, and we apply center-manifold reduction to obtain the amplitude equations for steady-state and Hopf bifurcation from the equilibrium state with a uniform phase distribution. When the population is described by a native frequency distribution that is reflection-symmetric about zero, the problem has circular symmetry. In the limit of zero extrinsic noise, although the critical eigenvalues are embedded in the continuous spectrum, the nonlinear coefficients in the amplitude equation remain finite in contrast to the singular behavior found in similar instabilities described by the Vlasov-Poisson equation. For a bimodal reflection-symmetric distribution, both types of bifurcation are possible and they coincide at a codimension-two Takens Bogdanov point. The steady-state bifurcation may be supercritical or subcritical and produces a time-independent synchronized state. The Hopf bifurcation produces both supercritical stable standing waves and supercritical unstable travelling waves. Previous work on the Hopf bifurcation in a bimodal population by Bonilla, Neu, and Spigler and Okuda and Kuramoto predicted stable travelling waves and stable standing waves, respectively. A comparison to these previous calculations shows that the prediction of stable travelling waves results from a failure to include all unstable modes.
\end{abstract}

keywords: oscillators, bifurcation, symmetry

October 28, 1993 


\section{Contents}

\begin{tabular}{lll}
\hline 1 & Introduction & 1
\end{tabular}

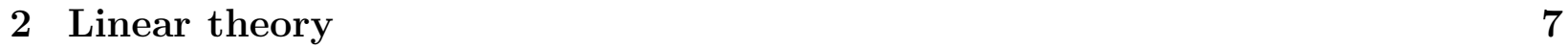

2.1 Simple roots and semisimple eigenvalues . . . . . . . . . . . . . . . . . . 10

$2.1 .1 \quad$ Real eigenvalues $\left(\operatorname{Re} z_{0}=0\right) \ldots \ldots \ldots \ldots 11$

2.1.2 Complex eigenvalues $\left(\operatorname{Re} z_{0} \neq 0\right) \ldots \ldots \ldots$. . . . . . . . . . 13

2.2 Multiple roots and non-semisimple eigenvalues . . . . . . . . . . . . . . . . . 14

2.3 Example of a bimodal population . . . . . . . . . . . . . . . . . . . . 18

3 Codimension-one bifurcations 20

3.1 Steady-state bifurcation . . . . . . . . . . . . . . . . . . . . . 21

3.1.1 Dynamics on the center manifold . . . . . . . . . . . . . . . . . . . . 22

3.1.2 Evaluation of $p_{\sigma}(0)$. . . . . . . . . . . . . . . . . . . . . . 24

3.1.3 Example of a bimodal population . . . . . . . . . . . . . . . . . 26

3.1.4 The $D \rightarrow 0$ limit . . . . . . . . . . . . . . . . . . . . . . . 27

3.1 .5 Perturbing $\mathrm{O}(2) \rightarrow \mathrm{SO}(2)$. . . . . . . . . . . . . . . . . . . . . . . 28

3.2 Hopf bifurcation . . . . . . . . . . . . . . . . . . . . . . . . . . . . 29

3.2 .1 Dynamics on the center manifold . . . . . . . . . . . . . . . . . . . . 30

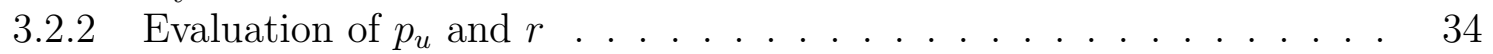

3.2 .3 Example of a bimodal population . . . . . . . . . . . . . . . . . . . 37

3.2 .4 Comparison to Bonilla, Neu, and Spiglen . . . . . . . . . . . . . . . . 39

3.2 .5 Comparison to Okuda and Kuramoto . . . . . . . . . . . . . . . . . . 39

3.2 .6 Perturbing $\mathrm{O}(2) \rightarrow \mathrm{SO}(2) \ldots \ldots \ldots$. . . . . . . . . . . . . . 41

\begin{tabular}{lll}
\hline 4 & Discussion & 41
\end{tabular}

\begin{tabular}{lll}
\hline 5 Acknowledgements & 42
\end{tabular}

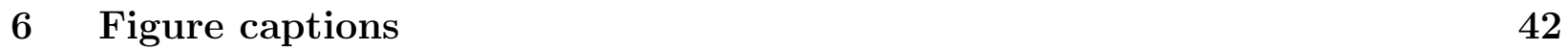

\section{Introduction}

The dynamics of a collection of weakly coupled limit cycle oscillators can be analyzed by treating the oscillator phases $\left\{\theta_{1}, \ldots, \theta_{N}\right\}$ as an autonomous dynamical system. In the context of biological oscillators this approach was advocated by Winfree and subsequently developed by Kuramoto for reaction-diffusion systems. [1, 2] In particular, Kuramoto formu- 
lated the widely studied model for phase dynamics:

$$
\dot{\theta}_{i}=\omega_{i}+\frac{K}{N} \sum_{j=1}^{N} \sin \left(\theta_{j}-\theta_{i}\right)+\xi_{i}(t)
$$

where the natural frequencies $\left\{\omega_{i}\right\}$ are randomly distributed according to a density $g(\omega)$. The effect of adding noise $\xi_{i}(t)$ to each phase was considered later by Sakaguchi. [3]

For large $N$, this model describes a transition to collective synchronized behavior as $K$ increases above a critical threshold $K_{c}$. More precisely, in terms of the order parameter,

$$
R(t) e^{i \psi(t)}=\frac{1}{N} \sum_{j=1}^{N} e^{i \theta_{j}(t)},
$$

one finds numerically a transition from an "incoherent" state with $R(t) \approx 0$ to a partially synchronized state with $R(t) \sim \sqrt{K-K_{c}}$ for $K>K_{c}$. For $g(\omega)$ chosen to be unimodal and symmetric about $\omega=0$, Kuramoto obtained an analytic expression for the order parameter $r$ as a function of the coupling $K$. His result agrees with numerical simulations and yields a simple expression for the critical coupling in the absence of noise

$$
K_{c}=\frac{2}{\pi g(0)}
$$

This transition has been analyzed by several authors 4 -11 and additional references can be found in Kuramoto and Nishikawa. [5] If $g(\omega)$ has compact support, then for sufficiently large $K$ there can be a transition to a completely synchronized state with $R \approx 1$. 112, 13

Since the incoherent state and the partially synchronized state emerge when the initial value problem is solved numerically, each must be stable in the appropriate range of $K$ at least in an operational sense. However the theoretical explanation of this stability has proved to be rather subtle even for the incoherent state, but there has been significant recent progress in the work of Strogatz and Mirollo. [7, 8] Following Sakaguchi, they considered the large $N$ limit of (11) and studied the Fokker-Planck equation

$$
\frac{\partial \rho}{\partial t}+\frac{\partial(\rho v)}{\partial \theta}=D \frac{\partial^{2} \rho}{\partial \theta^{2}}
$$


for the distribution of oscillators. The density $\rho(\theta, \omega, t)$ is defined so that $N g(\omega) \rho(\theta, \omega, t) d \theta d \omega$ describes the number of oscillators with natural frequencies in $[\omega, \omega+d \omega]$ and phases in $[\theta, \theta+d \theta]$. Thus $\rho(\theta, \omega, t) d \theta$ denotes the fraction of oscillators with natural frequency $\omega$ and phase in $[\theta, \theta+d \theta]$ and satisfies the normalization

$$
\int_{0}^{2 \pi} d \theta \rho(\theta, \omega, t)=1
$$

The identity

$$
R(t) \sin \left(\psi-\theta_{i}\right)=\frac{1}{N} \sum_{j=1}^{N} \sin \left(\theta_{j}-\theta_{i}\right)
$$

allows the phase velocity (1) of an oscillator to be written as

$$
v(\theta, \omega, t)=\omega+K R(t) \sin (\psi-\theta)
$$

and the order parameter (2) can be re-expressed in terms of the density

$$
R(t) e^{i \psi(t)}=\int_{0}^{2 \pi} d \theta \int_{-\infty}^{\infty} d \omega \rho(\theta, \omega, t) g(\omega) e^{i \theta} .
$$

The diffusion coefficient in (4) reflects the Gaussian noise terms $\xi_{i}(t)$ defined by

$$
\begin{aligned}
<\xi_{i}(t)> & =0 \\
<\xi_{i}(s) \xi_{j}(t)> & =2 D \delta_{i j} \delta(s-t) .
\end{aligned}
$$

Equations (4) and (7) - (8) provide a continuum description of the oscillator population for which issues of stability and bifurcation can be analyzed in some detail. The incoherent state is described by the uniform distribution $\rho_{0}=1 / 2 \pi$ and defines an equilibrium for (四) since $R=0$ at $\rho_{0}$. By defining $\eta$, the deviation from $\rho_{0}, \rho(\theta, \omega, t)=\rho_{0}+\eta(\theta, \omega, t)$ and eliminating $v$ in (4), the model can be rewritten as a single equation for $\eta$ :

$$
\frac{\partial \eta}{\partial t}=\mathcal{L} \eta+\mathcal{N}(\eta)
$$


where

$$
\mathcal{L} \eta \equiv D \frac{\partial^{2} \eta}{\partial \theta^{2}}-\omega \frac{\partial \eta}{\partial \theta}+\frac{K \rho_{0}}{2}\left[e^{-i \theta} \int_{0}^{2 \pi} d \theta^{\prime} \int_{-\infty}^{\infty} d \omega^{\prime} \eta\left(\theta^{\prime}, \omega^{\prime}, t\right) g\left(\omega^{\prime}\right) e^{i \theta^{\prime}}+c c\right]
$$

and the nonlinear terms are

$$
\begin{aligned}
\mathcal{N}(\eta)=\frac{K}{2}\{ & e^{i \theta}\left(\eta-i \frac{\partial \eta}{\partial \theta}\right) \int_{0}^{2 \pi} d \theta^{\prime} \int_{-\infty}^{\infty} d \omega^{\prime} \eta\left(\theta^{\prime}, \omega^{\prime}, t\right) g\left(\omega^{\prime}\right) e^{-i \theta^{\prime}} \\
& \left.+e^{-i \theta}\left(\eta+i \frac{\partial \eta}{\partial \theta}\right) \int_{0}^{2 \pi} d \theta^{\prime} \int_{-\infty}^{\infty} d \omega^{\prime} \eta\left(\theta^{\prime}, \omega^{\prime}, t\right) g\left(\omega^{\prime}\right) e^{i \theta^{\prime}}\right\} .
\end{aligned}
$$

Note that the normalization (5) of $\rho$ implies

$$
\int_{0}^{2 \pi} d \theta \eta(\theta, \omega, t)=0
$$

An important qualitative feature of this evolution equation is its symmetry. The group $\mathrm{O}(2)$ is generated by rotations

$$
\phi \cdot(\theta, \omega)=(\theta+\phi, \omega)
$$

and reflections

$$
\kappa \cdot(\theta, \omega)=-(\theta, \omega)
$$

which act on functions $\eta(\theta, \omega)$ in the usual way:

$$
(\gamma \cdot \eta)(\theta, \omega)=\eta\left(\gamma^{-1} \cdot(\theta, \omega)\right) \quad \gamma \in \mathrm{O}(2)
$$

Provided $g(\omega)$ satisfies

$$
g(\omega)=g(-\omega)
$$

the equation for $\eta(11)$ has $\mathrm{O}(2)$ symmetry; more precisely $\gamma \cdot(\mathcal{L} \eta)=\mathcal{L}(\gamma \cdot \eta)$ and $\gamma \cdot \mathcal{N}(\eta)=$ $\mathcal{N}(\gamma \cdot \eta)$ for all $\gamma \in \mathrm{O}(2)$. A realistic population of oscillators will not have zero mean frequency as implied by (18), but if their frequency distribution $G\left(\omega_{\text {lab }}\right)$ is symmetric about the mean $\bar{\omega}$ then in the rotating frame defined by

$$
(\theta, \omega)=\left(\theta_{\mathrm{lab}}-\bar{\omega} t, \omega_{\mathrm{lab}}-\bar{\omega}\right)
$$


the frequency distribution $g(\omega)=G(\omega+\bar{\omega})$ will satisfy (18). The densities in the lab frame and in the rotating frame are related by円

$$
\rho(\theta, \omega, t)=\rho_{\mathrm{lab}}(\theta+\bar{\omega} t, \omega+\bar{\omega}, t)
$$

If $g$ lacks reflectional invariance (18) then the symmetry in the rotating frame is reduced to the rotations $\mathrm{SO}(2) \cdot[14]$

Strogatz and Mirollo provide a linear stability analysis of $\rho_{0}$ focussing especially on populations with even distributions (18) that decrease monotonically away from $\omega=0$. [7] They show that $\mathcal{L}$ has a continuous spectrum with real part equal to $-D$, and that it may also have point spectrum (eigenvalues) depending on the coupling strength. For $K$ sufficiently small, the point spectrum is empty, but as the coupling increases a real eigenvalue $\lambda$ emerges from the continuous spectrum and for $K>K_{c}$ moves into the right half plane $\lambda>0$ signifying linear instability of $\rho_{0}$. The condition $\lambda=0$ determines a critical coupling

$$
K_{c}=2\left[\int_{-\infty}^{\infty} \frac{D g(\omega) d \omega}{D^{2}+\omega^{2}}\right]^{-1}
$$

that agrees with results for $K_{c}$ derived by Sakaguchi using different arguments and reduces to (3) when $D \rightarrow 0$. Thus the onset of synchronization is shown to coincide with the appearance of a linearly unstable mode for the Fokker-Planck equilibrium. Subsequently Strogatz et al. demonstrated that for $D=0$ and $K<K_{c}$, the linearized equation predicts $\rho_{0}$ is stable in the sense that $R(t)$ decays via a "phase mixing" mechanism that is qualitatively similar to Landau damping in a collisionless plasma. 8

Recently Bonilla, Neu, and Spigler investigated bifurcations from $\rho_{0}$ assuming a reflectionsymmetric frequency distribution (18) but relaxing the requirement that $g(\omega)$ have a single peak. [1] For bimodal distributions they showed that $\mathcal{L}$ could have complex eigenvalues so

\footnotetext{
${ }^{1}$ In the lab frame the evolution equation for $\rho_{\text {lab }}\left(\theta_{\text {lab }}, \omega_{\text {lab }}, t\right)$ has rotation symmetry $\operatorname{SO}(2)$ but not reflection symmetry. Calculations done using lab frame variables have puzzling features that are non-generic for $\mathrm{SO}(2)$ symmetry and have their origin in the full $\mathrm{O}(2)$ symmetry of the rotating frame.
} 
that the instability of $\rho_{0}$ occurs via Hopf bifurcation. Amplitude equations were derived to describe the nonlinear development of the instabilities, but unfortunately the Hopf analysis retains only half of the unstable eigenvectors and as a result nonlinear travelling and standing wave solutions were overlooked. The fact that the $\mathrm{O}(2)$ symmetry leads to degenerate eigenvalues of multiplicity-two was apparently not recognized.

Independently, Okuda and Kuramoto analyzed the onset of collective behavior in a population comprised of two sub-populations using a different model; each sub-population had a unique frequency in the absence of coupling and was described by a number density. [9] The instability of the incoherent state was studied under several assumptions on the couplings between the oscillators. In the regime where the coupling between the populations equals the coupling within a population, their model is a special case of (凷. They also find that instability can arise from either real or complex eigenvalues and derived amplitude equations for the nonlinear behavior. Their equations include both travelling and standing wave solutions although the role of symmetry in the problem is not discussed.

In this paper I re-examine the bifurcation problem and derive amplitude equations for both steady state and Hopf bifurcation using center manifold reduction. In Section II, the spectrum of $\mathcal{L}$ is discussed to clarify the treatment of complex eigenvalues and to discuss the occurrence of non-semi-simple real eigenvalues. These arise naturally at the multi-critical point where the Hopf and steady-state bifurcations coalesce. This codimension-two point corresponds to a so-called Takens-Bogdanov bifurcation and the relevant amplitude equations will be investigated in future work. The discussion of Section II is facilitated by the similarities of $\mathcal{L}$ to the linear operator for the Vlasov-Poisson equation which has been discussed elsewhere. [15]

In Section III, the amplitude equations are derived and their limiting form as $D \rightarrow 0$ determined. The results are strikingly different from analogous amplitude equations for 
Vlasov instabilities; in the collisionless plasma case the coefficients diverge as the critical eigenvalues approach the continuum but in this model the coefficients have finite limits. [16] In the nonlinear theory, the presence of $\mathrm{O}(2)$ symmetry is exploited to take advantage of the well-established theory of bifurcation in systems with circular symmetry. [17, [18] For a reflection-symmetric bimodal population comprised of two Lorentzians, we evaluate the cubic normal form coefficients. For the steady-state bifurcation the appearance of the synchronized state can be either supercritical or subcritical. In the Hopf bifurcation we find that the standing waves are supercritical and stable while the travelling waves are supercritical and unstable. These conclusions are compared to previous studies. [9, 11]

\section{Linear theory}

The Fourier expansion of $\eta$,

$$
\eta(\theta, \omega)=\sum_{l=-\infty}^{\infty} \eta_{l}(\omega) e^{i l \theta}
$$

allows the linear operator (12) to be re-expressed as

$$
\mathcal{L} \eta=\sum_{l=-\infty}^{\infty}\left(\mathrm{L}_{l} \eta_{l}\right) e^{i l \theta}
$$

where

$$
\mathrm{L}_{l} \eta_{l}=-i l(\omega-i l D) \eta_{l}+\pi \rho_{0} K\left(\delta_{l, 1}+\delta_{l,-1}\right) \int_{-\infty}^{\infty} d \omega^{\prime} g\left(\omega^{\prime}\right) \eta_{l}\left(\omega^{\prime}\right) .
$$

Note that the normalization condition (14) on $\eta$ implies

$$
\eta_{l=0} \equiv 0
$$

An adjoint operator $(A, \mathcal{L} B) \equiv\left(\mathcal{L}^{\dagger} A, B\right)$ can be defined for the inner product

$$
(A, B) \equiv \int_{0}^{2 \pi} d \theta \int_{-\infty}^{\infty} d \omega A(\theta, \omega)^{*} B(\theta, \omega) .
$$


In terms of the Fourier expansion $A=\sum A_{l} e^{i l \theta}$, this definition yields

$$
\mathcal{L}^{\dagger} A=\sum_{l=-\infty}^{\infty}\left(\mathrm{L}_{l}^{\dagger} A_{l}\right) e^{i l \theta}
$$

where

$$
\left(\mathrm{L}_{l}^{\dagger} A_{l}\right)(\omega)=i l(\omega+i l D) A_{l}(\omega)+\frac{g(\omega) K}{2}\left(\delta_{l, 1}+\delta_{l,-1}\right) \int_{-\infty}^{\infty} d \omega^{\prime} A_{l}\left(\omega^{\prime}\right) .
$$

It is convenient to introduce a concise notation for the integration over $\omega$ in (26)

$$
<A, B>\equiv \int_{-\infty}^{\infty} d \omega A^{*} B
$$

then $\mathrm{L}_{l}$ and $\mathrm{L}_{l}^{\dagger}$ satisfy $<A, \mathrm{~L}_{l} B>=<\mathrm{L}_{l}^{\dagger} A, B>$.

The eigenvalue problem for $\mathcal{L}$,

$$
\mathcal{L} \Psi=\lambda \Psi
$$

can be treated on each Fourier subspace separately so we set

$$
\Psi(\theta, \omega)=e^{i l \theta} \psi(\omega)
$$

and reduce $(30)$ to

$$
\mathrm{L}_{l} \psi=\lambda \psi
$$

After scaling the eigenvalue by $\lambda=-i l z$, the eigenvalue equation (32) becomes

$$
[(\omega-z)-i l D] \psi=\frac{-i l K}{2}\left(\delta_{l, 1}+\delta_{l,-1}\right) \int_{-\infty}^{\infty} d \omega^{\prime} g\left(\omega^{\prime}\right) \psi\left(\omega^{\prime}\right)
$$

The first observation is that $|l|=1$ is a necessary condition for $\psi$ to be a well-behaved eigenfunction. [7] When $|l| \neq 1$ then (33) becomes $[(\omega-z)-i l D] \psi=0$, and we have $\psi \equiv 0$ unless $\operatorname{Im} z=-l D$. For $\operatorname{Im} z=-l D$ then $[(\omega-z)-i l D]$ is non-zero except at $\omega=\operatorname{Re} z$, so the only solutions are delta functions $\delta(\omega-\operatorname{Re} z)$. These solutions correspond to a continuous spectrum for $\mathrm{L}_{l}$ along $\operatorname{Re} \lambda=l \operatorname{Im} z=-l^{2} D$; as $D \rightarrow 0$ the continuous spectrum moves onto 
the imaginary axis. For $|l| \neq 1$ this is the only contribution to the spectrum of $\mathcal{L}$ and it does not depend on the coupling $K$ or on the frequency distribution $g(\omega)$.

For $|l|=1$ it is possible to have eigenfunctions that are non-singular; their occurrence does depend on $K$ and $g(\omega)$. Now the eigenvalue equation (33) becomes

$$
[(\omega-z)-i l D] \psi=\frac{-i l K}{2} \int_{-\infty}^{\infty} d \omega^{\prime} g\left(\omega^{\prime}\right) \psi\left(\omega^{\prime}\right) . \quad(|l|=1)
$$

When $\operatorname{Im} z=-l D$, then as before we get singular solutions corresponding to a continuous spectrum along $\operatorname{Re} \lambda=-D$; the detailed form of these solutions will not be needed. If $\operatorname{Im} z \neq-l D$ then $(\omega-z)-i l D$ cannot vanish, and we have

$$
\psi=\frac{-i l K / 2}{(\omega-z)-i l D} \int_{-\infty}^{\infty} d \omega^{\prime} g\left(\omega^{\prime}\right) \psi\left(\omega^{\prime}\right) .
$$

If the integral vanishes then $\psi \equiv 0$ so we may assume the integral is non-zero and normalize $\psi$ such that

$$
\int_{-\infty}^{\infty} d \omega^{\prime} g\left(\omega^{\prime}\right) \psi\left(\omega^{\prime}\right)=1
$$

Consistency between (35) and (36) requires $z$ to be a root of the "spectral function"

$$
\Lambda_{l}(z) \equiv 1+\frac{i l K}{2} \int_{-\infty}^{\infty} d \omega^{\prime} \frac{g\left(\omega^{\prime}\right)}{\omega^{\prime}-z-i l D} . \quad(|l|=1) .
$$

The spectral function $\Lambda_{l}$ satisfies two identities; for arbitrary $g(\omega)$ we have

$$
\Lambda_{l}(z)^{*}=\Lambda_{-l}\left(z^{*}\right)
$$

so when $\Lambda_{l}\left(z_{0}\right)=0$ determines an eigenvalue $\lambda=-i l z_{0}$ then $\Lambda_{-l}\left(z_{0}^{*}\right)=0$ determines the complex-conjugate eigenvalue $\lambda^{*}$. In addition when the frequency distribution is reflectionsymmetric (18), the problem has $O(2)$ symmetry and $\Lambda_{l}$ satisfies

$$
\Lambda_{l}(z)^{*}=\Lambda_{l}\left(-z^{*}\right)
$$

Now roots come in pairs whenever $\operatorname{Re} z \neq 0$, i.e. whenever $\lambda$ is complex. 
The roots of $\Lambda_{l}(z)$ determine the eigenvalues and the nature of the root determines whether the eigenvalue is semisimple or not. Recall that the projection operator $P_{\lambda}$ onto the generalized eigenspace $E_{\lambda}$ for an isolated eigenvalue $\lambda=-i l z_{0}$ is defined by a contour integral, 119

$$
P_{\lambda} A=\frac{1}{2 \pi i} \int_{\Gamma_{0}} d \lambda^{\prime}\left(\lambda^{\prime}-\mathcal{L}\right)^{-1} A ;
$$

where $\Gamma_{0}$ is a small loop enclosing $\lambda$ in a counter-clockwise sense. The resolvent operator $(\lambda-\mathcal{L})^{-1}$ can be expressed as $\mathbb{Z}^{2}$

$$
(\lambda-\mathcal{L})^{-1} A=\sum_{l=-\infty}^{\infty}\left(R_{l}(\lambda) A_{l}\right) e^{i l \theta}
$$

where $R_{l}(\lambda) \equiv\left(\lambda-\mathrm{L}_{l}\right)^{-1}$ denotes the resolvent for $\mathrm{L}_{l}$ and can be calculated explicitly

$$
R_{l}(\lambda) A_{l}=\frac{A_{l}(\omega)}{i l \omega+l^{2} D+\lambda}+\frac{\left(\delta_{l, 1}+\delta_{l,-1}\right) K / 2}{\Lambda_{l}(i l \lambda)\left(i l \omega+l^{2} D+\lambda\right)} \int_{-\infty}^{\infty} d \omega^{\prime} \frac{g\left(\omega^{\prime}\right) A_{l}\left(\omega^{\prime}\right)}{i l \omega^{\prime}+l^{2} D+\lambda} .
$$

With (41) $P_{\lambda}$ may be simplified to

$$
P_{\lambda} A=\frac{e^{i \theta}}{2 \pi i} \int_{\Gamma_{0}} d \lambda^{\prime} R_{1}\left(\lambda^{\prime}\right) A_{1}+\frac{e^{-i \theta}}{2 \pi i} \int_{\Gamma_{0}} d \lambda^{\prime} R_{-1}\left(\lambda^{\prime}\right) A_{-1}
$$

since eigenvalues can only occur for $|l|=1$. If $E_{\lambda}$, the range of $P_{\lambda}$, is one-dimensional then $\lambda$ is simple; when the dimension is greater than one and the eigenvectors of $\mathcal{L}$ span $E_{\lambda}$ the eigenvalue is semisimple. If $\operatorname{dim} E_{\lambda}>1$, and the eigenvectors do not span $E_{\lambda}$, then $\lambda$ is non-semisimple. Simple roots of $\Lambda_{l}(z)$ lead to simple or semisimple eigenvalues and multiple roots give eigenvalues that are non-semisimple. We discuss this point in detail for the case of a reflection-symmetric frequency distribution and $\mathrm{O}(2)$ symmetry.

\subsection{Simple roots and semisimple eigenvalues}

For a simple root $z_{0}$ of $\Lambda_{1}(z)$, we have

$$
\Lambda_{1}\left(z_{0}\right)=0 \quad \Lambda_{1}^{\prime}\left(z_{0}\right) \neq 0
$$

\footnotetext{
${ }^{2}$ In this sum, the term $l=0$ may be omitted since the relevant space of functions satisfies (25).
} 
where $\Lambda^{\prime}$ denotes the derivative of $\Lambda_{1}\left(z_{0}\right)$ with respect to $z$. The identities (38) - (39) imply the additional simple roots

$$
\Lambda_{1}\left(-z_{0}^{*}\right)=0 \quad \Lambda_{-1}\left(z_{0}^{*}\right)=0 \quad \Lambda_{-1}\left(-z_{0}\right)=0 .
$$

If $\operatorname{Re} z_{0}=0$ ( a real eigenvalue) then $z_{0}$ and $-z_{0}^{*}$ are the same root and $\operatorname{roots}\left(z_{0}^{*},-z_{0}\right)$ also coincide. When $\operatorname{Re} z_{0} \neq 0$ (complex eigenvalues) then these roots are all distinct.

\subsubsection{Real eigenvalues $\left(\operatorname{Re} z_{0}=0\right)$}

With $\operatorname{Re} z_{0}=0$, the roots $z_{0}=$ and $z_{0}^{*}$ for $l= \pm 1$ lead to linearly independent eigenvectors for the real eigenvalue $\lambda=-i z_{0}$. From (31) and (35), these vectors are

$$
\Psi(\theta, \omega)=e^{i \theta} \psi(\omega)
$$

where

$$
\psi(\omega)=\frac{-i K / 2}{\left(\omega-z_{0}\right)-i D}
$$

and the conjugate function

$$
\Psi(\theta, \omega)^{*}=e^{-i \theta} \psi(\omega)^{*}
$$

Note, that these eigenvectors are related by reflection $\Psi(\theta, \omega)^{*}=(\kappa \cdot \Psi)(\theta, \omega)$. Corresponding to $\Psi$ and $\kappa \cdot \Psi$, there are adjoint eigenvectors

$$
\begin{aligned}
\mathcal{L}^{\dagger} \tilde{\Psi} & =\lambda \tilde{\Psi} \\
\mathcal{L}^{\dagger}(\kappa \cdot \tilde{\Psi}) & =\lambda(\kappa \cdot \tilde{\Psi})
\end{aligned}
$$

where

$$
\tilde{\Psi}(\theta, \omega)=\frac{e^{i \theta}}{2 \pi} \tilde{\psi}(\omega)
$$

with

$$
\tilde{\psi}(\omega)=\frac{-g(\omega)}{\Lambda_{1}^{\prime}\left(z_{0}\right)^{*}\left(\omega-z_{0}^{*}+i D\right)} .
$$


These eigenvectors are conjugate $\kappa \cdot \tilde{\Psi}=\tilde{\Psi}^{*}$ and satisfy

$$
\begin{aligned}
(\tilde{\Psi}, \Psi) & =(\kappa \cdot \tilde{\Psi}, \kappa \cdot \Psi)=1 \\
(\tilde{\Psi}, \kappa \cdot \Psi) & =(\kappa \cdot \tilde{\Psi}, \Psi)=0 .
\end{aligned}
$$

For example, the eigenvalue equation (49) can be verified directly from the definitions (27) $-(28):$

$$
\begin{aligned}
& \mathcal{L}^{\dagger} \tilde{\Psi}=\frac{e^{i \theta}}{2 \pi} \mathrm{L}_{1}^{\dagger} \tilde{\psi}(\omega) \\
& =\frac{e^{i \theta}}{2 \pi \Lambda_{1}^{\prime}\left(z_{0}\right)^{*}}\left[-\frac{i g(\omega)}{\left(\omega-z_{0}^{*}+i D\right)}\right. \\
& \left.-\frac{g(\omega) K}{2} \int_{-\infty}^{\infty} d \omega^{\prime} \frac{g\left(\omega^{\prime}\right)}{\left(\omega^{\prime}-z_{0}^{*}+i D\right)}\right] \\
& =\frac{e^{i \theta}}{2 \pi \Lambda_{1}^{\prime}\left(z_{0}\right)^{*}}[-i g(\omega) \\
& \left.-\frac{i g(\omega) z_{0}^{*}}{\left(\omega-z_{0}^{*}+i D\right)}-i g(\omega)\left(\Lambda_{-1}\left(z_{0}^{*}\right)-1\right)\right] \\
& =i z_{0}^{*} \frac{e^{i \theta}}{2 \pi \Lambda_{1}^{\prime}\left(z_{0}\right)^{*}}\left(\frac{-g(\omega) z_{0}^{*}}{\left(\omega-z_{0}^{*}+i D\right)}\right) \\
& =\lambda \tilde{\Psi}
\end{aligned}
$$

since $\lambda=\lambda^{*}=-i z_{0}$.

Since $\Lambda_{1}\left(z_{0}\right)=0$ corresponds to a simple pole, the result of projecting $\eta$ onto $\Psi$ is easily evaluated. From (42) - (43) we have

$$
\begin{aligned}
P_{\lambda} \eta= & \frac{e^{i \theta}}{2 \pi i} \int_{\Gamma_{0}} d \lambda^{\prime} R_{1}\left(\lambda^{\prime}\right) \eta_{1}+\frac{e^{-i \theta}}{2 \pi i} \int_{\Gamma_{0}} d \lambda^{\prime} R_{-1}\left(\lambda^{\prime}\right) \eta_{-1} \\
= & \frac{e^{i \theta}}{2 \pi i} \int_{\Gamma_{0}} d \lambda^{\prime} \frac{K / 2}{\Lambda_{1}\left(i \lambda^{\prime}\right)\left(i \omega+D+\lambda^{\prime}\right)} \int_{-\infty}^{\infty} d \omega^{\prime} \frac{g\left(\omega^{\prime}\right) \eta_{1}\left(\omega^{\prime}\right)}{i \omega^{\prime}+D+\lambda^{\prime}} \\
& +\frac{e^{-i \theta}}{2 \pi i} \int_{\Gamma_{0}} d \lambda^{\prime} \frac{K / 2}{\Lambda_{-1}\left(-i \lambda^{\prime}\right)\left(-i \omega+D+\lambda^{\prime}\right)} \int_{-\infty}^{\infty} d \omega^{\prime} \frac{g\left(\omega^{\prime}\right) \eta_{-1}\left(\omega^{\prime}\right)}{-i \omega^{\prime}+D+\lambda^{\prime}}
\end{aligned}
$$




$$
\begin{aligned}
= & e^{i \theta} \frac{K / 2}{i \Lambda_{1}^{\prime}\left(z_{0}\right)\left(i \omega+D-i z_{0}\right)} \int_{-\infty}^{\infty} d \omega^{\prime} \frac{g\left(\omega^{\prime}\right) \eta_{1}\left(\omega^{\prime}\right)}{i \omega^{\prime}+D-i z_{0}} \\
& +e^{-i \theta} \frac{K / 2}{-i \Lambda_{-1}^{\prime}\left(z_{0}^{*}\right)\left(-i \omega+D+i z_{0}^{*}\right)} \int_{-\infty}^{\infty} d \omega^{\prime} \frac{g\left(\omega^{\prime}\right) \eta_{-1}\left(\omega^{\prime}\right)}{-i \omega^{\prime}+D+i z_{0}^{*}} \\
= & e^{i \theta} \psi(\omega)<\tilde{\psi}, \eta_{1}>+e^{-i \theta} \psi(\omega)^{*}<\tilde{\psi}^{*}, \eta_{-1}> \\
= & (\tilde{\Psi}, \eta) \Psi(\theta, \omega)+(\kappa \cdot \tilde{\Psi}, \eta) \kappa \cdot \Psi(\theta, \omega) .
\end{aligned}
$$

The eigenvectors $\Psi$ and $\kappa \cdot \Psi$ clearly span the range of $P_{\lambda}$.

\subsubsection{Complex eigenvalues $\left(\operatorname{Re} z_{0} \neq 0\right)$}

When $\operatorname{Re} z_{0} \neq 0$, then $z_{0}$ and $-z_{0}$ are distinct roots of $\Lambda_{1}(z)$ and $\Lambda_{-1}(z)$, respectively, that determine two linearly independent eigenvectors for the complex eigenvalue $\lambda=-i z_{0}$. These eigenvectors are $\Psi$ given by (46) - (47) and the reflected function

$$
\kappa \cdot \Psi=e^{-i \theta} \psi(-\omega)
$$

This second eigenvector was omitted in the Hopf analysis of Bonilla et al. [11] The remaining roots $-z_{0}^{*}$ and $z_{0}^{*}$ for $\Lambda_{1}$ and $\Lambda_{-1}$ determine the conjugate eigenvectors $\Psi^{*}$ and $(\kappa \cdot \Psi)^{*}$ corresponding to the conjugate eigenvalue $\lambda^{*}=i z_{0}^{*}$. The adjoint eigenvectors $\tilde{\Psi}$ and $\kappa \cdot \tilde{\Psi}$ have the same form (51) - (52), however now (49) - (50) become

$$
\begin{aligned}
\mathcal{L}^{\dagger} \tilde{\Psi} & =\lambda^{*} \tilde{\Psi} \\
\mathcal{L}^{\dagger}(\kappa \cdot \tilde{\Psi}) & =\lambda^{*}(\kappa \cdot \tilde{\Psi})
\end{aligned}
$$

the adjoint eigenvectors for $\lambda$ are the conjugate functions $\tilde{\Psi}^{*}$ and $(\kappa \cdot \tilde{\Psi})^{*}$. The orthogonality relations (53) - (54) remain valid.

The projection $P_{\lambda} \eta$ can be evaluated as before (56) except that now the relevant root (pole) for $l=-1$ is $\Lambda_{-1}\left(-z_{0}\right)=0$. The final result has the same form

$$
P_{\lambda} \eta=(\tilde{\Psi}, \eta) \Psi+(\kappa \cdot \tilde{\Psi}, \eta) \kappa \cdot \Psi
$$


A similar evaluation of $P_{\lambda^{*}} \eta$ gives

$$
P_{\lambda^{*}} \eta=\left(P_{\lambda} \eta\right)^{*}
$$

In each case the eigenvalue is semisimple with multiplicity-two.

\subsection{Multiple roots and non-semisimple eigenvalues}

A single root, $\Lambda_{1}\left(z_{0}\right)=0$, determines exactly one eigenvector (46) - (47) for the eigenvalue $\lambda=-i z_{0}$. There can be additional linearly independent eigenvectors for the same eigenvalue $\lambda$ only if $\Lambda_{-1}$ simultaneously has a root at $-z_{0}$. In any event, a given eigenvalue can never have more than two eigenvectors. However if the root $z_{0}$ of $\Lambda_{1}$ is not simple, the range of $P_{\lambda}$ turns out to have dimension greater than the number of eigenvectors because there are generalized eigenvectors in addition to the true eigenvectors. A generalized eigenvector $\Upsilon$ satisfies

$$
\begin{aligned}
(\mathcal{L}-\lambda)^{j} \Upsilon & =0 \\
(\mathcal{L}-\lambda)^{j-1} \Upsilon & \neq 0
\end{aligned}
$$

for some integer $j \geq 2$. Note that (62) simply says that $(\mathcal{L}-\lambda)^{j-1} \Upsilon$ is an eigenvector for $\lambda$.

Since degenerate roots of $\Lambda_{1}$ arise when simple roots collide, we expect generalized eigenvectors to enter the linear problem whenever eigenvalues collide as parameters are varied. The connection between degenerate roots and generalized eigenvalues can be made explicit as follows. Consider the simplest situation $j=2$ and let $\Psi=e^{i \theta} \psi$ be a true eigenvector corresponding to $\Lambda_{1}\left(z_{0}\right)=0$. We seek a generalized eigenvector $\Upsilon=e^{i \theta} v$ satisfying

$$
\begin{aligned}
& (\mathcal{L}-\lambda) \Upsilon=\Psi \\
& (\mathcal{L}-\lambda)^{2} \Upsilon=0
\end{aligned}
$$


note that (64) implies (65). From the definition of $\mathcal{L}$, (64) can be rewritten as

$$
\left(\omega-z_{0}-i D\right) v+\frac{i K}{2} \int_{-\infty}^{\infty} d \omega^{\prime} g\left(\omega^{\prime}\right) v\left(\omega^{\prime}\right)=\frac{K / 2}{\omega-z_{0}-i D}
$$

upon multiplying by $g(\omega) /\left(\omega-z_{0}-i D\right)$ and integrating (66) becomes

$$
\Lambda_{1}\left(z_{0}\right) \int_{-\infty}^{\infty} d \omega^{\prime} g\left(\omega^{\prime}\right) v\left(\omega^{\prime}\right)=-i \Lambda_{1}^{\prime}\left(z_{0}\right)
$$

Since $\Lambda_{1}\left(z_{0}\right)=0$, the assumption (64) that a generalized eigenvector $\Upsilon$ exists leads immediately to a contradiction if the root is simple, i.e. if $\Lambda_{1}^{\prime}\left(z_{0}\right) \neq 0$. An extension of this argument shows that a generalized eigenvector with $j \geq 2$ for $\lambda$ cannot occur unless the first $j-1$ derivatives of $\Lambda_{1}$ vanish at the root.

Bonilla et al. found that the transition between a bimodal distribution $g(\omega)$ supporting real eigenvalues and a bimodal distribution $g(\omega)$ supporting complex eigenvalues occurs via a non-semisimple real eigenvalue corresponding to a double root:

$$
\Lambda_{1}\left(z_{0}\right)=0 \quad \Lambda_{1}^{\prime}\left(z_{0}\right)=0 \quad \Lambda_{1}^{\prime \prime}\left(z_{0}\right) \neq 0
$$

where $\operatorname{Re} z_{0}=0$. For more complicated distributions, it is likely that double roots with Re $z_{0} \neq 0$ are also possible. The case of a real eigenvalue is considered here and we assume $\operatorname{Re} z_{0}=0$ for the remainder of this section. The double root (68) at $z_{0}$ then implies a double root at $-z_{0}$ for $\Lambda_{-1}$ :

$$
\Lambda_{-1}\left(-z_{0}\right)=0 \quad \Lambda_{-1}^{\prime}\left(-z_{0}\right)=0 \quad \Lambda_{-1}^{\prime \prime}\left(-z_{0}\right) \neq 0 .
$$

In applications with double roots, the frequency distribution will typically depend on at least one additional parameter $\omega_{0}$, for example in a bimodal distribution $\omega_{0}$ could be the distance between peaks in $g\left(\omega, \omega_{0}\right)$. Then $\Lambda_{1}^{\prime}=0$ determines $z_{0}$ as a function of $K$ and $\omega_{0}$ and $\Lambda_{1}=0$ implies a relation $K=K\left(\omega_{0}\right)$ between the parameters. The eigenvector 
corresponding to $\Lambda_{1}\left(z_{0}\right)=0$ is then $\Psi=e^{i \theta} \psi$ with

$$
\psi(\omega)=\frac{-i K\left(\omega_{0}\right) / 2}{\left(\omega-z_{0}\right)-i D}
$$

and the generalized eigenvector $\Upsilon=e^{i \theta} v$ is determined by 66

$$
v(\omega)=\frac{K\left(\omega_{0}\right) / 2}{\left(\omega-z_{0}-i D\right)^{2}} .
$$

One can always add to $v(\omega)$ an arbitrary multiple of the homogeneous solution $\psi$ but without loss of generality we have set the coefficient of this term to zero. Reflection by $\kappa$ gives the eigenvector and generalized eigenvector corresponding to the second $\operatorname{root}(69): \kappa \cdot \Psi=$ $e^{-i \theta} \psi(-\omega)$ and $\kappa \cdot \Upsilon=e^{-i \theta} v(-\omega)$.

For the adjoint eigenvalue problem, there is an eigenvector $\left(\mathcal{L}^{\dagger}-\lambda\right) \tilde{\Psi}=0$ and generalized eigenvector $\left(\mathcal{L}^{\dagger}-\lambda\right) \tilde{\Upsilon}=\tilde{\Psi}$ given by $\tilde{\Psi}=e^{i \theta} \tilde{\psi}(\omega)$ and $\tilde{\Upsilon}=e^{i \theta} \tilde{v}(\omega)$ where

$$
\begin{aligned}
\tilde{\psi}(\omega) & =\left[\frac{-2 g\left(\omega, \omega_{0}\right)}{\Lambda_{1}^{\prime \prime}\left(z_{0}\right)\left(\omega-z_{0}-i D\right)^{2}}+\frac{2 \Lambda_{1}^{\prime \prime \prime}\left(z_{0}\right) g\left(\omega, \omega_{0}\right)}{3 \Lambda_{1}^{\prime \prime}\left(z_{0}\right)^{2}\left(\omega-z_{0}-i D\right)}\right]^{*} \\
\tilde{v}(\omega) & =\left[\frac{2 i g\left(\omega, \omega_{0}\right)}{\Lambda_{1}^{\prime \prime}\left(z_{0}\right)\left(\omega-z_{0}-i D\right)}\right]^{*} .
\end{aligned}
$$

In addition the reflected vectors $\kappa \cdot \tilde{\Psi}$ and $\kappa \cdot \tilde{\Upsilon}$ solve the same equations. These eigenvectors satisfy the biorthogonality relations

$$
\begin{array}{ll}
(\tilde{\Psi}, \Psi)=<\tilde{\psi}, \psi>=1 & (\kappa \cdot \tilde{\Psi}, \kappa \cdot \Psi)=<\kappa \cdot \tilde{\psi}, \kappa \cdot \psi>=1 \\
(\tilde{\Upsilon}, \Upsilon)=<\tilde{v}, v>=1 & (\kappa \cdot \tilde{\Upsilon}, \kappa \cdot \Upsilon)=<\kappa \cdot \tilde{v}, \kappa \cdot v>=1
\end{array}
$$

and

$$
\begin{array}{ll}
(\tilde{\Psi}, \Upsilon)=<\tilde{\psi}, v>=0 & (\kappa \cdot \tilde{\Psi}, \kappa \cdot \Upsilon)=0 \\
(\tilde{\Upsilon}, \Psi)=<\tilde{v}, \psi>=0 & (\kappa \cdot \tilde{\Upsilon}, \kappa \cdot \Psi)=0
\end{array}
$$


The evaluation of $P_{\lambda} \eta$ is now more involved because the poles are not simple; from (43) we have

$$
\begin{aligned}
P_{\lambda} \eta= & \frac{e^{i \theta}}{2 \pi i} \int_{\Gamma_{0}} d \lambda^{\prime} R_{1}\left(\lambda^{\prime}\right) \eta_{1}+\frac{e^{-i \theta}}{2 \pi i} \int_{\Gamma_{0}} d \lambda^{\prime} R_{-1}\left(\lambda^{\prime}\right) \eta_{-1} \\
= & \frac{e^{i \theta}}{2 \pi i} \int_{\Gamma_{0}} d \lambda^{\prime} \frac{K / 2}{\Lambda_{1}\left(i \lambda^{\prime}\right)\left(i \omega+D+\lambda^{\prime}\right)} \int_{-\infty}^{\infty} d \omega^{\prime} \frac{g\left(\omega^{\prime}, \omega_{0}\right) \eta_{1}\left(\omega^{\prime}\right)}{i \omega^{\prime}+D+\lambda^{\prime}} \\
& +\frac{e^{-i \theta}}{2 \pi i} \int_{\Gamma_{0}} d \lambda^{\prime} \frac{K / 2}{\Lambda_{-1}\left(-i \lambda^{\prime}\right)\left(-i \omega+D+\lambda^{\prime}\right)} \int_{-\infty}^{\infty} d \omega^{\prime} \frac{g\left(\omega^{\prime}, \omega_{0}\right) \eta_{-1}\left(\omega^{\prime}\right)}{-i \omega^{\prime}+D+\lambda^{\prime}} .
\end{aligned}
$$

Consider the first integral for $K=K\left(\omega_{0}\right)$ when $\Lambda_{1}$ has a double root; the contribution from this double pole is

$$
\begin{aligned}
& \frac{e^{i \theta}}{2 \pi i} \int_{\Gamma_{0}} d \lambda^{\prime} \frac{K\left(\omega_{0}\right) / 2}{\Lambda_{1}\left(i \lambda^{\prime}\right)\left(i \omega+D+\lambda^{\prime}\right)} \int_{-\infty}^{\infty} d \omega^{\prime} \frac{g\left(\omega^{\prime}, \omega_{0}\right) \eta_{1}\left(\omega^{\prime}\right)}{i \omega^{\prime}+D+\lambda^{\prime}} \\
& \quad=e^{i \theta} \frac{d}{d \lambda^{\prime}}\left[\frac{\left(\lambda^{\prime}-\lambda\right)^{2} K\left(\omega_{0}\right) / 2}{\Lambda_{1}\left(i \lambda^{\prime}\right)\left(i \omega+D+\lambda^{\prime}\right)}\left\{\int_{-\infty}^{\infty} d \omega^{\prime} \frac{g\left(\omega^{\prime}, \omega_{0}\right) \eta_{1}\left(\omega^{\prime}\right)}{i \omega^{\prime}+D+\lambda^{\prime}}\right\}\right]_{\lambda^{\prime}=\lambda}
\end{aligned}
$$

Expanding $\Lambda_{1}$

$$
\Lambda_{1}\left(i \lambda^{\prime}\right)=i^{2} \Lambda_{1}^{\prime \prime}(i \lambda) \frac{\left(\lambda^{\prime}-\lambda\right)^{2}}{2}+i^{3} \Lambda_{1}^{\prime \prime \prime}(i \lambda) \frac{\left(\lambda^{\prime}-\lambda\right)^{3}}{3 !}+\cdots,
$$

and evaluating the right-hand side of (81) gives

$$
\frac{e^{i \theta}}{2 \pi i} \int_{\Gamma_{0}} d \lambda^{\prime} \frac{K\left(\omega_{0}\right) / 2}{\Lambda_{1}\left(i \lambda^{\prime}\right)\left(i \omega+D+\lambda^{\prime}\right)} \int_{-\infty}^{\infty} d \omega^{\prime} \frac{g\left(\omega^{\prime}, \omega_{0}\right) \eta_{1}\left(\omega^{\prime}\right)}{i \omega^{\prime}+D+\lambda^{\prime}}=(\tilde{\Psi}, \eta) \Psi+(\tilde{\Upsilon}, \eta) \Upsilon
$$

A similar evaluation of the second integral in (『9) yields $(\tilde{\Psi}, \eta) \Psi+(\tilde{\Upsilon}, \eta) \Upsilon$ which is the complex conjugate of (83). Overall we have

$$
P_{\lambda} \eta=(\tilde{\Psi}, \eta) \Psi+(\tilde{\Upsilon}, \eta) \Upsilon+(\tilde{\Psi}, \eta) \Psi+(\tilde{\Upsilon}, \eta) \Upsilon
$$

thus a purely imaginary double root determines a real eigenvalue with two eigenvectors and two generalized eigenvectors. 
Complete expansions in terms of the eigenfunctions of $\mathrm{L}_{l}$ and hence $\mathcal{L}$ can be derived as in the Vlasov case 15 if desired. We do not need these expansions for the bifurcation analysis, it is sufficient to know how to project out the components of $\eta$ along the critical eigenvectors in the three cases of interest: semisimple real, semisimple complex, and non-semisimple real.

\subsection{Example of a bimodal population}

We illustrate this discussion for the population

$$
g\left(\omega, \omega_{0}\right)=\frac{\epsilon}{2 \pi}\left[\frac{1}{\left(\omega+\omega_{0}\right)^{2}+\epsilon^{2}}+\frac{1}{\left(\omega-\omega_{0}\right)^{2}+\epsilon^{2}}\right]
$$

comprised of two sub-populations centered at $\omega= \pm \omega_{0}$; the $\epsilon \rightarrow 0$ limit yields the delta function distribution considered independently by Okuda and Kuramoto 9 and by Bonilla et al. [11] When $\omega_{0}^{2}>\epsilon^{2} / 3$, there are two peaks in $g\left(\omega, \omega_{0}\right)$ located at

$$
\left(\frac{\omega_{\text {peak }}}{\omega_{0}}\right)^{2}=\left[2-\sqrt{1+\left(\epsilon / \omega_{0}\right)^{2}}\right] \sqrt{1+\left(\epsilon / \omega_{0}\right)^{2}} .
$$

For $\operatorname{Im} z>-D$, the evaluation of $\Lambda_{1}$ yields

$$
\Lambda_{1}(z)=\frac{[z+i(\epsilon+D)]^{2}-\omega_{0}^{2}-i K[z+i(\epsilon+D)] / 2}{[z+i(\epsilon+D)]^{2}-\omega_{0}^{2}} .
$$

which has two roots for $\omega_{0}>0$ :

$$
z_{ \pm}=i\left[(K / 4)-\epsilon-D \pm \sqrt{(K / 4)^{2}-\omega_{0}^{2}}\right]
$$

At $\omega_{0}=0$, the expression in (87) has only a single root $z_{+}=i[(K / 2)-\epsilon-D]$; the solution $z_{-}$at $-i(\epsilon+D)$ is an artifact. For $0<4 \omega_{0}<K$, the roots are pure imaginary and the eigenvalues are real; along the line $4 \omega_{0}=K$ the roots collide, a double root forms, and a non-semisimple real eigenvalue occurs. When $4 \omega_{0}>K$, the eigenvalues are complex and Hopf bifurcation is possible.

\footnotetext{
${ }^{3}$ This case is easily treated by closing the integration contour in the lower half plane. One can show that for $\operatorname{Im} z<-D$ and $K \geq 0$, the spectral function has no roots.
} 
For $4 \omega_{0}<K$, the root $z_{+}$in (88) determines an eigenvalue if $\operatorname{Im} z_{+}>-D$ which requires either $K>2\left(\omega_{0}^{2}+\epsilon^{2}\right) / \epsilon$ or $K>4 \epsilon$; the solution $z_{-}$determines an eigenvalue when $4 \epsilon<K<2\left(\omega_{0}^{2}+\epsilon^{2}\right) / \epsilon$. The eigenvalues $\lambda_{ \pm}=-i z_{ \pm}$disappear into the continuous spectrum at $-D$ when $\lambda_{ \pm}=-D$. This defines a boundary in parameter space

$$
K=K_{e} \equiv 2\left(\omega_{0}^{2}+\epsilon^{2}\right) / \epsilon
$$

which is tangent to $K=4 \omega_{0}$ at $\omega_{0}=\epsilon+D$. The real eigenvalues cross the imaginary axis $\lambda_{ \pm}=0$ along a second boundary

$$
K=K_{c} \equiv 2\left[\omega_{0}^{2}+\left(\epsilon^{2}+D^{2}\right)\right] /(\epsilon+D)
$$

with $\lambda_{+}=0$ if $K<4(\epsilon+D)$ and $\lambda_{-}=0$ if $K>4(\epsilon+D)$. This second boundary is tangent to $K=4 \omega_{0}$ at $K=4(\epsilon+D)$ where we find a non-semisimple eigenvalue at zero; here the steady state bifurcation turns into Hopf bifurcation. Figure 1 shows the $\left(K, \omega_{0}\right)$ parameter space with the curves corresponding to eigenvalues coalescing with the continuum, crossing the imaginary axis, or colliding on the real axis.

For $4 \omega_{0}>K$, the roots (88) are complex and the eigenvalues $\left(\lambda, \lambda^{*}\right)$ are given by

$$
\lambda=-i z_{0}=\left(K-K_{h}\right) / 4+i \Omega
$$

where $\Omega \equiv \sqrt{\omega_{0}^{2}-(K / 4)^{2}}$ and $K_{h} \equiv 4(\epsilon+D)$. If $K$ decreases below $K_{e}^{\prime} \equiv 4 \epsilon$ the eigenvalues $\left(\lambda, \lambda^{*}\right)$ disappear into the continuous spectrum at $\operatorname{Re} \lambda=-D$; as $K$ increases above $K_{h}$ there is a Hopf bifurcation. The curves $K=K_{e}^{\prime}$ and $K=K_{h}$ are also shown in Fig. 1.

In an early discussion of bimodal populations without extrinsic noise $D=0$, Kuramoto suggested that once the maximum of $g(\omega)$ was no longer at the average frequency (here $\bar{\omega}=0)$ the incoherent state would go unstable for some $K=K_{c 0}<K_{c}$ with synchronized behavior nucleating among oscillators whose frequencies lay near the maxima of $g(\omega)$. [2] Although the development of bimodal peaks in (85) for $\omega_{0}>\sqrt{\epsilon} / 3$, does alter the stability 
properties of the incoherent state; the effects are somewhat more complicated. As we shall see below in our analysis of the steady state bifurcation, for

$$
(\epsilon+D) \sqrt{\frac{\epsilon+2 D}{3 \epsilon+4 D}}<\omega_{0}<(\epsilon+D),
$$

the incoherent state is stable when $K<K_{c}$ and there is a subcritical steady state bifurcation to a synchronized state at $K=K_{c}$. There is no prior onset of synchronization in the peaks of the distribution. The further qualitative change in the stability properties of the incoherent state with increasing $\omega_{0}$ above $(\epsilon+D)$ is to replace the steady state bifurcation by a Hopf bifurcation which leads to time dependent behavior (cf. Section 3.2) in the form

of stable nonlinear "standing waves". These waves appear to be the type of state Kuramoto envisioned; they are discussed in greater detail below. It seems likely that these conclusions remain true in the limit $D \rightarrow 0$.

\section{Codimension-one bifurcations}

For the nonlinear analysis to follow we require the Fourier expansion of $\mathcal{N}(\eta)$. With

$$
e^{i \theta}\left(\eta-i \frac{\partial \eta}{\partial \theta}\right)=\sum_{l \neq 0,1} l \eta_{l-1}(\omega) e^{i l \theta}
$$

and

$$
\int_{0}^{2 \pi} d \theta^{\prime} \int_{-\infty}^{\infty} d \omega^{\prime} \eta\left(\theta^{\prime}, \omega^{\prime}, t\right) g\left(\omega^{\prime}\right) e^{-i \theta^{\prime}}=2 \pi<g, \eta_{1}>,
$$

$\mathcal{N}(\eta)$ in (13) becomes

$$
\begin{aligned}
\mathcal{N}(\eta)=-\pi K & \left\{e^{i \theta}<g, \eta_{-1}>\eta_{2}+e^{-i \theta}<g, \eta_{1}>\eta_{-2}\right. \\
& \left.+\sum_{|l| \geq 2} l e^{i l \theta}\left[<g, \eta_{-1}>\eta_{l+1}(\omega)-<g, \eta_{1}>\eta_{l-1}(\omega)\right]\right\} .
\end{aligned}
$$




\subsection{Steady-state bifurcation}

We consider a simple pure imaginary root $\Lambda_{1}\left(z_{0}\right)=0$ so that $\lambda=-i z_{0}$ is real. As a parameter such as $K$ is varied, we assume that $\lambda$ crosses through $\lambda=0$ at $K=K_{c}$. From (46) - (48) there are two eigenfunctions $\Psi$ and $\kappa \cdot \Psi=\Psi^{*}$, with amplitudes $\left(\alpha, \alpha^{*}\right)$ defined by $\alpha(t) \equiv(\tilde{\Psi}, \eta)$. The distribution $\eta$ decomposes into the critical linear modes $P_{\lambda} \eta=$ $\alpha(t) \Psi(\theta, \omega)+c c$, and the remaining degrees of freedom $S(\theta, \omega) \equiv \eta-P_{\lambda} \eta$. Thus we have

$$
\eta(\theta, \omega, t)=[\alpha(t) \Psi(\theta, \omega)+c c]+S(\theta, \omega, t)
$$

where $(\tilde{\Psi}, S)=0$. In terms of the Fourier components of $\eta$ and $S$, we have

$$
\eta_{l}=\alpha \psi \delta_{l, 1}+\alpha^{*} \psi^{*} \delta_{l,-1}+S_{l}
$$

note that the normalization (25) requires

$$
S_{l=0}=0 .
$$

By projecting with $\tilde{\Psi}$, the evolution equation (11) can be rewritten as

$$
\begin{aligned}
\dot{\alpha} & =\lambda \alpha+(\tilde{\Psi}, \mathcal{N}(\eta)) \\
\frac{\partial S}{\partial t} & =\mathcal{L} S+\{\mathcal{N}(\eta)-[(\tilde{\Psi}, \mathcal{N}(\eta)) \Psi+c c]\}
\end{aligned}
$$

since $<g, \eta_{l}>=\alpha \delta_{l, 1}+\alpha^{*} \delta_{l,-1}+<g, S_{l}>$ in (95), the Fourier decomposition of these equations gives

$$
\begin{aligned}
\dot{\alpha}=\lambda \alpha-\pi & K\left(\alpha^{*}+<g, S_{-1}>\right)<\tilde{\psi}, S_{2}> \\
\frac{\partial S}{\partial t}=\mathcal{L} S-\pi & \left\{\left\{e^{i \theta}\left(\alpha^{*}+<g, S_{-1}>\right)\left(S_{2}-<\tilde{\psi}, S_{2}>\psi\right)\right.\right. \\
+ & 2 e^{i 2 \theta}\left[\left(\alpha^{*}+<g, S_{-1}>\right) S_{3}(\omega)-\left(\alpha+<g, S_{1}>\right)\left(\alpha \psi+S_{1}\right)\right] \\
+ & \sum_{l \geq 3} l e^{i l \theta}\left[\left(\alpha^{*}+<g, S_{-1}>\right) S_{l+1}(\omega)-\left(\alpha+<g, S_{1}>\right) S_{l-1}(\omega)\right] \\
& +c c\} .
\end{aligned}
$$




\subsubsection{Dynamics on the center manifold}

The equations (101)-(102) are obviously coupled but on the center manifold the "transverse" degrees of freedom $S$ depend on time through the critical amplitudes; this may be expressed in terms of a function $H\left(\theta, \omega, \alpha, \alpha^{*}\right)$ :

$$
S(\theta, \omega, t)=H\left(\theta, \omega, \alpha(t), \alpha(t)^{*}\right)=\sum_{l=-\infty}^{\infty} H_{l}\left(\theta, \alpha(t), \alpha(t)^{*}\right) e^{i l \theta} .
$$

In geometric terms, $H$ describes the graph of the center manifold near the incoherent state. 20, 21, 22 Thus solutions on the center manifold satisfy (101) - (102) with $S$ replaced by $H$

$$
\begin{aligned}
& \dot{\alpha}=\lambda \alpha-\pi K\left(\alpha^{*}+<g, H_{-1}>\right)<\tilde{\psi}, H_{2}> \\
& \frac{\partial S}{\partial t}=\mathcal{L} H-\pi K\left\{e^{i \theta}\left(\alpha^{*}+<g, H_{-1}>\right)\left(H_{2}-<\tilde{\psi}, H_{2}>\psi\right)\right. \\
&+ 2 e^{i 2 \theta}\left[\left(\alpha^{*}+<g, H_{-1}>\right) H_{3}(\omega)-\left(\alpha+<g, H_{1}>\right)\left(\alpha \psi+H_{1}\right)\right] \\
&+\sum_{l \geq 3} l e^{i l \theta}\left[\left(\alpha^{*}+<g, H_{-1}>\right) H_{l+1}(\omega)-\left(\alpha+<g, H_{1}>\right) H_{l-1}(\omega)\right] \\
&+c c\}
\end{aligned}
$$

the equation (104) for the mode amplitude now decouples from $S$ and defines an autonomous two-dimensional system.

The center manifold dynamics (104) and the graph function are both constrained by the $\mathrm{O}(2)$ symmetry of the problem. The Fourier coefficients of $H$ for $l>0$ must have the form

$$
H_{l}\left(\omega, \alpha, \alpha^{*}\right)=\alpha^{l} h_{l}(\omega, \sigma)
$$

where $h_{l}(\omega, \sigma)$ satisfies

$$
h_{l}(-\omega, \sigma)^{*}=h_{l}(\omega, \sigma)
$$

and $\sigma=|\alpha|^{2}$ denotes the basic $\mathrm{O}(2)$ invariant. Similarly (104), the equation for $\alpha$ must have the general form

$$
\dot{\alpha}=p(\sigma) \alpha
$$


where $p(\sigma)$ is a real-valued function of a single variable. For small $\alpha$, the leading terms in (104) are therefore

$$
\dot{\alpha}=\alpha\left[p(0)+p_{\sigma}(0)|\alpha|^{2}+\cdots\right]
$$

and comparison to (104) shows $p(0)=\lambda$ and $p_{\sigma}(0)$ remains to be calculated.

We briefly summarize the derivation of (106) - (108). The form of $H$ is constrained by the fact that the center manifold is mapped to itself $\mathrm{O}(2)$ transformations. [23] More precisely, if $\eta(\theta, \omega)$ corresponds to a point on the center manifold and $\gamma \in \mathrm{O}(2)$, then the transformed function $\gamma \cdot \eta$ will also lie on the center manifold. When we represent elements of the center manifold using (103),

$$
\eta(\theta, \omega)=\alpha \Psi(\theta, \omega)+\alpha^{*} \Psi^{*}(\theta, \omega)+H\left(\theta, \omega, \alpha, \alpha^{*}\right)
$$

then $\gamma \cdot \eta$ must also be of this form

$$
(\gamma \cdot \eta)(\theta, \omega)=\alpha^{\prime} \Psi(\theta, \omega)+\alpha^{\prime *} \Psi^{*}(\theta, \omega)+H\left(\theta, \omega, \alpha^{\prime}, \alpha^{\prime *}\right)
$$

for some transformed amplitude $\alpha^{\prime}$. The constraints on $H$ implied by this requirement follow from considering the generators of $\mathrm{O}(2)$ in $(15)$ - (16). In conjunction with (17) we find for rotations

$$
[\alpha \Psi(\theta-\phi, \omega)+c c]+H\left(\theta-\phi, \omega, \alpha, \alpha^{*}\right)=\left[\alpha e^{-i \phi} \Psi(\theta, \omega)+c c\right]+H\left(\theta, \omega, e^{-i \phi} \alpha, e^{i \phi} \alpha^{*}\right)
$$

and

$$
[\alpha \Psi(-\theta,-\omega)+c c]+H\left(-\theta,-\omega, \alpha, \alpha^{*}\right)=\left[\alpha^{*} \Psi(\theta, \omega)+c c\right]+H\left(\theta, \omega, \alpha^{*}, \alpha\right)
$$

for reflection. Since $\Psi(\theta-\phi, \omega)=e^{-i \phi} \Psi(\theta, \omega)$ and $\Psi(-\theta,-\omega)=\Psi(\theta, \omega)^{*}$, the amplitude $\alpha$ transforms under $\mathrm{O}(2)$ by

$$
\begin{aligned}
\phi \cdot \alpha & =e^{-i \phi} \alpha \\
\kappa \cdot \alpha & =\alpha^{*}
\end{aligned}
$$


and $H$ must satisfy

$$
\begin{aligned}
H\left(\theta-\phi, \omega, \alpha, \alpha^{*}\right) & =H\left(\theta, \omega, e^{-i \phi} \alpha, e^{i \phi} \alpha^{*}\right) \\
H\left(-\theta,-\omega, \alpha, \alpha^{*}\right) & =H\left(\theta, \omega, \alpha^{*}, \alpha\right) .
\end{aligned}
$$

Restated for the Fourier coefficients $H_{l}$, these relations require

$$
e^{-i l \phi} H_{l}\left(\omega, \alpha, \alpha^{*}\right)=H_{l}\left(\theta, \omega, e^{-i \phi} \alpha, e^{i \phi} \alpha^{*}\right)
$$

and

$$
H_{l}\left(-\omega, \alpha, \alpha^{*}\right)^{*}=H_{l}\left(\omega, \alpha^{*}, \alpha\right)
$$

respectively. The rotational constraint (118) states that the function $\left(\alpha^{*}\right)^{l} H_{l}$ is invariant under rotations, hence it depends on the mode amplitudes $\left(\alpha, \alpha^{*}\right)$ only through the basic invariant $\sigma=|\alpha|^{2}$ and must contain an overall factor of $\left(\alpha^{*}\right)^{l}$ :

$$
\left(\alpha^{*}\right)^{l} H_{l}\left(\omega, \alpha, \alpha^{*}\right)=\sigma^{l} h_{l}(\omega, \sigma)
$$

where $h_{l}(\omega, \sigma)$ is an arbitrary function of $\omega$ and $\sigma$. Dividing by $\left(\alpha^{*}\right)^{l}$ gives the form of $H_{l}$ in (106); the reflection symmetry (119) then further requires that $h_{l}(\omega, \sigma)$ satisfy (107).

The $\mathrm{O}(2)$ invariance of the center manifold also implies that the vector field in (104) must commute with the representation of $\mathrm{O}(2)$ given in $(114)$ - (115). This can be explicitly verified using the properties of $H_{l}$ in (118) - (119). Any smooth two-dimensional vector field that commutes with this representation must have the form (108); see for example Golubitsky, Stewart, and Schaeffer, chapter XII, §5.

\subsubsection{Evaluation of $p_{\sigma}(0)$}

From (106), $H_{1}$ and $H_{2}$ have the form

$$
\begin{aligned}
& H_{1}=\alpha h_{1}(\omega, \sigma)=\alpha\left[h_{1}^{(0)}(\omega)+\mathcal{O}(\sigma)\right] \\
& H_{2}=\alpha^{2} h_{2}(\omega, \sigma)=\alpha^{2}\left[h_{2}^{(2)}(\omega)+\mathcal{O}(\sigma)\right],
\end{aligned}
$$

\footnotetext{
${ }^{4}$ cf. Golubitsky, Stewart, and Schaeffer, chapter XII, $\S 4$
} 
however the geometry of the center manifold requires that the lowest order terms be quadratic in $\left(\alpha, \alpha^{*}\right)$ so $h_{1}^{(0)}=0$; hence the center manifold vector field (104) becomes

$$
\begin{aligned}
\dot{\alpha} & =\lambda \alpha-\pi K \alpha|\alpha|^{2}\left(1+<g, h_{1}^{*}>\right)<\tilde{\psi}, h_{2}> \\
& =\alpha\left[\lambda-\pi K<\tilde{\psi}, h_{2}^{(2)}>|\alpha|^{2}+\cdots\right] .
\end{aligned}
$$

Comparing (109) and (124) we obtain $p_{\sigma}(0)$

$$
p_{\sigma}(0)=-\pi K<\tilde{\psi}, h_{2}^{(2)}>
$$

in terms of the center manifold coefficient $h_{2}^{(2)}$.

When $S$ is given by (103) we have

$$
\frac{\partial S}{\partial t}=\frac{\partial H}{\partial \alpha} \dot{\alpha}+\frac{\partial H}{\partial \alpha^{*}} \dot{\alpha}^{*}
$$

for solutions on the center manifold, and consistency with (102) requires

$$
\begin{aligned}
& \frac{\partial H}{\partial \alpha} \dot{\alpha}+\frac{\partial H}{\partial \alpha^{*}} \dot{\alpha}^{*}=\mathcal{L} H- K\left\{\left\{e^{i \theta}\left(\alpha^{*}+<g, H_{-1}>\right)\left(H_{2}-<\tilde{\psi}, H_{2}>\psi\right)\right.\right. \\
&+2 e^{i 2 \theta}\left[\left(\alpha^{*}+<g, H_{-1}>\right) H_{3}(\omega)-\left(\alpha+<g, H_{1}>\right)\left(\alpha \psi+H_{1}\right)\right] \\
&+ \sum_{l \geq 3} l e^{i l \theta}\left[\left(\alpha^{*}+<g, H_{-1}>\right) H_{l+1}(\omega)-\left(\alpha+<g, H_{1}>\right) H_{l-1}(\omega)\right] \\
&+c c\}
\end{aligned}
$$

where $\dot{\alpha}$ is given by (104). This provides the equation needed to determine the expansion coefficients of $H$; for small $|\alpha|$, we can solve (127) using a power series expansion in $\left(\alpha, \alpha^{*}\right)$. Separating the different Fourier components of (127) for $l=1, l=2$ and $l \geq 3$, we find

$$
\begin{aligned}
& \frac{\partial H_{1}}{\partial \alpha} \dot{\alpha}+\frac{\partial H_{1}}{\partial \alpha^{*}} \dot{\alpha}^{*}=\mathrm{L}_{1} H_{1}-\pi K\left(\alpha^{*}+<g, H_{-1}>\right)\left(H_{2}-<\tilde{\psi}, H_{2}>\psi\right) \\
& \frac{\partial H_{2}}{\partial \alpha} \dot{\alpha}+\frac{\partial H_{2}}{\partial \alpha^{*}} \dot{\alpha}^{*}=\mathrm{L}_{2} H_{2}-2 \pi K\left[\left(\alpha^{*}+<g, H_{-1}>\right) H_{3}(\omega)\right.
\end{aligned}
$$




$$
\begin{array}{r}
\left.-\left(\alpha+<g, H_{1}>\right)\left(\alpha \psi+H_{1}\right)\right] \\
\frac{\partial H_{l}}{\partial \alpha} \dot{\alpha}+\frac{\partial H_{l}}{\partial \alpha^{*}} \dot{\alpha}^{*}=\mathrm{L}_{l} H_{l}-l \pi K\left[\left(\alpha^{*}+<g, H_{-1}>\right) H_{l+1}(\omega)\right. \\
\left.-\left(\alpha+<g, H_{1}>\right) H_{l-1}(\omega)\right]
\end{array}
$$

respectively, and inspecting these equations shows that the inhomogeneous term $\alpha^{2} \psi$ in (129) that forces a non-zero term $h_{2}^{(2)}(\omega)$ at the lowest order term in $H_{2}$. Since $h_{1}^{(0)}(\omega)=0$, the balance at second order in (129) is $2 \lambda h_{2}^{(2)}=\mathrm{L}_{2} h_{2}^{(2)}+2 \pi K \psi$ which gives

$$
h_{2}^{(2)}(\omega)=\frac{-i \pi K \psi(\omega)}{\omega-z_{0}-i 2 D}
$$

Thus the cubic coefficient in the center manifold dynamics (125) is given by

$$
p_{\sigma}(0)=\frac{-\pi^{2} K^{3}}{2 \Lambda_{1}^{\prime}\left(z_{0}\right)} \int_{-\infty}^{\infty} d \omega \frac{g(\omega)}{\left(\omega-z_{0}-i D\right)^{2}\left(\omega-z_{0}-i 2 D\right)}
$$

Note that $p_{\sigma}(0)$ is real as expected from $\mathrm{O}(2)$ symmetry.

\subsubsection{Example of a bimodal population}

For the population in (85) the first steady state bifurcation occurs when the eigenvalue $\lambda_{+}$crosses zero; this occurs along $K=K_{c}$ Fig. 1 . Along this steady state locus, the coefficient (132) can be easily evaluated by closing the contour in the lower half plane:

$$
p_{\sigma}(0)=\frac{\pi^{2} K_{c}^{2}(3 \epsilon+4 D)\left(\omega_{0}^{2}-\omega_{d}^{2}\right)}{2\left[(\epsilon+D)^{2}-\omega_{0}^{2}\right]\left[(\epsilon+2 D)^{2}+\omega_{0}^{2}\right]}
$$

where

$$
\omega_{d} \equiv(\epsilon+D) \sqrt{\frac{\epsilon+2 D}{3 \epsilon+4 D}} .
$$

Since $\omega_{0}<(\epsilon+D)$ when this bifurcation occurs, the overall sign of $p_{\sigma}(0)$ is determined by $\omega_{0}^{2}-\omega_{d}^{2}$. For $\omega_{0}<\omega_{d}$, the bifurcation is supercritical to a stable synchronized state: for 
$\omega_{0}>\omega_{d}$, we have $p_{\sigma}(0)>0$ and the bifurcation is subcritical. Note that $p_{\sigma}(0)$ is finite in the limit $D \rightarrow 0$ as long as $\epsilon>0$; this feature is examined in greater generality below.

For $\epsilon>0$ and $D=0$, the transition from supercritical to subcritical bifurcation was noted by Kuramoto, 22 and at $\epsilon=0$ and $D>0$ it was found by Bonilla et al. and Okuda and Kuramoto.[11, 9] The former group also calculated the fifth order term necessary to determine that the subcritical branch turns around at a saddle node bifurcation and becomes stable at finite amplitude.

\subsubsection{The $D \rightarrow 0$ limit}

If $D=0$ then the critical eigenvalues emerge from the continuous spectrum simultaneously with the onset of the linear instability, and it is of interest to examine the behaviour of the amplitude equation in this regime. At criticality for either steady-state or Hopf bifurcation $\operatorname{Re} \lambda=0$ so $\operatorname{Im} z_{0}=0$, thus we consider the behavior of $p_{\sigma}(0)$ when $D \rightarrow 0$ with $z_{0}=0$. The integrand in (132) has poles at $\omega=i D$ and $\omega=i 2 D$ that approach the contour of integration as $D \rightarrow 0$. We assume $g(\omega)$ is analytic at $\omega=0$ so that the contour can be deformed slightly below the axis and the limits evaluated using the Plemej formulas.

It is convenient to first consider the limiting behavior of $\Lambda_{l}$. From (37) we re-express the derivatives of $\Lambda_{l}(z)$ by integrating by parts

$$
\frac{\partial^{n}}{\partial z^{n}} \Lambda_{l}(z)=\delta_{n, 0}+\frac{i l K}{2} \int_{-\infty}^{\infty} d \omega \frac{\partial^{n} g(\omega) / \partial \omega^{n}}{(\omega-z-i l D)}
$$

thus when $z=r$ is real-valued, then as $D \rightarrow 0$

$$
\lim _{D \rightarrow 0} \frac{\partial^{n}}{\partial z^{n}} \Lambda_{1}(r)=\delta_{n, 0}+\frac{i K}{2}\left[\mathcal{P} \int_{-\infty}^{\infty} d \omega \frac{\partial^{n} g(\omega) / \partial \omega^{n}}{\omega-r}+i \pi \frac{\partial^{n} g(r)}{\partial \omega^{n}}\right]
$$

where $\mathcal{P}$ denotes a principal value integral. For steady-state bifurcation, when $g(\omega)$ is even in $\omega$ and $r=0$ at $K=K_{c}$, these limits simplify:

$$
\lim _{D \rightarrow 0} \frac{\partial^{n}}{\partial z^{n}} \Lambda_{1}(0)=i K_{c} \int_{0}^{\infty} d \omega \frac{\partial^{n} g(\omega) / \partial \omega^{n}}{\omega} \quad n \text { odd }
$$




$$
\lim _{D \rightarrow 0} \frac{\partial^{n}}{\partial z^{n}} \Lambda_{1}(0)=\delta_{n, 0}+\frac{-\pi K_{c}}{2} \frac{\partial^{n} g(0)}{\partial \omega^{n}} \quad n \text { even }
$$

Note that for $n=0$ by setting $\Lambda_{1}(0)=0$ in (138) recovers the previous result (3) for $K_{c}=2 / \pi g(0)$.

For small $D$ the behavior of $p_{\sigma}(0)$ is extracted in the same way; setting $z_{0}=0$ we find

$$
\begin{aligned}
p_{\sigma}(0) & =\frac{-\pi^{2} K_{c}^{3}}{2 \Lambda_{1}^{\prime}(0)} \lim _{D \rightarrow 0} \int_{-\infty}^{\infty} d \omega \frac{g(\omega)}{(\omega-i D)^{2}(\omega-i 2 D)} \\
& =i\left(\pi K_{c}\right)^{2} \frac{\Lambda_{1}^{\prime \prime}(0)}{2 \Lambda_{1}^{\prime}(0)}+\mathcal{O}(D) \\
& =\frac{-\pi}{g(0)^{2}} \frac{\partial^{2} g(0)}{\partial \omega^{2}}\left[\int_{0}^{\infty} d \omega \frac{\partial g(\omega) / \partial \omega}{\omega}\right]^{-1}+\mathcal{O}(D) .
\end{aligned}
$$

Once differences in notation are taken into account, this result agrees with the coefficient obtained by Kuramoto by the "self-consistent" method. [2] Notably absent is the kind of singular behavior found in the Vlasov amplitude equations in the corresponding limit. [16] For a monotonic reflection-symmetric profile this gives $p_{\sigma}(0)<0$ in the limit of weak diffusion. Thus at the level of the amplitude equation, we expect supercritical bifurcation to a stable synchronized state in this regime. However when $D=0$ center manifold theory no longer justifies our reduction to two dimensions; the qualitative agreement at $D=0$ between numerical simulations [4] and our amplitude equation may be fortuitous.

\subsubsection{Perturbing $\mathrm{O}(2) \rightarrow \mathrm{SO}(2)$}

If the distribution $g(\omega)$ is perturbed so that the reflection symmetry (18) is broken, then the bifurcation problem (in the rotating frame (19)) has only the rotational symmetry $\mathrm{SO}(2)$. In this case one expects the real eigenvalue of multiplicity-two to split into a non-degenerate complex conjugate pair and the steady state bifurcation considered here to be perturbed to a Hopf bifurcation. The resulting Hopf bifurcation leads to time-periodic states in the 
form of rotating or travelling waves; Knobloch discusses such perturbed bifurcations in more detail. [14

\subsection{Hopf bifurcation}

We next consider a simple complex root $\Lambda_{1}\left(z_{0}\right)=0$ so that $z_{0} \neq-z_{0}^{*}$ and $\lambda=-i z_{0}$ is complex. As $K$ is varied through $K_{h}$,we assume that $\operatorname{Re} \lambda$ crosses through $\operatorname{Re} \lambda=0$ at $K=K_{h}$. From (57) there are two eigenfunctions $\Psi$ and $\kappa \cdot \Psi=\Psi^{*}$, with amplitudes $(\alpha, \beta)$ defined by $\alpha(t) \equiv(\tilde{\Psi}, \eta)$ and $\beta(t) \equiv(\kappa \cdot \tilde{\Psi}, \eta)$. With the projections (60) - (61), the distribution $\eta$ decomposes as before,

$$
\eta(\theta, \omega, t)=[\alpha(t) \Psi(\theta, \omega)+\beta(t) \kappa \cdot \Psi(\theta, \omega)+c c]+S(\theta, \omega, t)
$$

where $(\tilde{\Psi}, S)=(\kappa \cdot \tilde{\Psi}, S)=0$.

Projecting (11) with $\tilde{\Psi}$ and $\kappa \cdot \tilde{\Psi}$ yields

$$
\begin{aligned}
\dot{\alpha} & =\lambda \alpha+(\tilde{\Psi}, \mathcal{N}(\eta)) \\
\dot{\beta} & =\lambda \beta+(\kappa \cdot \tilde{\Psi}, \mathcal{N}(\eta)) \\
\frac{\partial S}{\partial t} & =\mathcal{L} S+\{\mathcal{N}(\eta)-[\Psi(\tilde{\Psi}, \mathcal{N}(\eta))+\kappa \cdot \Psi(\kappa \cdot \tilde{\Psi}, \mathcal{N}(\eta))+c c]\} .
\end{aligned}
$$

In terms of the Fourier components of $\eta_{l}$

$$
\begin{aligned}
\eta_{l} & =\left(\alpha \psi+\beta^{*} \kappa \cdot \psi^{*}\right) \delta_{l, 1}+\left(\alpha^{*} \psi^{*}+\beta \kappa \cdot \psi\right) \delta_{l,-1}+S_{l} \\
<g, \eta_{l}> & =\left(\alpha+\beta^{*}\right) \delta_{l, 1}+\left(\alpha^{*}+\beta\right) \delta_{l,-1}+<g, S_{l}>,
\end{aligned}
$$

and the expansion of $\mathcal{N}(\eta)$ in (95), (141)-(143) can be re-written as

$$
\begin{aligned}
\dot{\alpha} & =\lambda \alpha-\pi K\left(\alpha^{*}+\beta+<g, S_{-1}>\right)<\tilde{\psi}, S_{2}> \\
\dot{\beta} & =\lambda \beta-\pi K\left(\alpha+\beta^{*}+<g, S_{1}>\right)<\kappa \cdot \tilde{\psi}, S_{-2}> \\
\frac{\partial S}{\partial t} & =\mathcal{L} S-\pi K\left\{e^{i \theta}\left(\alpha^{*}+\beta+<g, S_{-1}>\right)\left[S_{2}-<\tilde{\psi}, S_{2}>\psi-<\kappa \cdot \tilde{\psi}^{*}, S_{2}>\kappa \cdot \psi^{*}\right]\right.
\end{aligned}
$$




$$
\begin{aligned}
& +2 e^{i 2 \theta}\left[\left(\alpha^{*}+\beta+<g, S_{-1}>\right) S_{3}(\omega)-\left(\alpha+\beta^{*}+<g, S_{1}>\right)\left(\alpha \psi+\beta^{*} \kappa \cdot \psi^{*}+S_{1}\right)\right] \\
& +\sum_{l \geq 3} l e^{i l \theta}\left[\left(\alpha^{*}+\beta+<g, S_{-1}>\right) S_{l+1}(\omega)-\left(\alpha+\beta^{*}+<g, S_{1}>\right) S_{l-1}(\omega)\right] \\
& +c c\} .
\end{aligned}
$$

\subsubsection{Dynamics on the center manifold}

For a solution on the center manifold near the incoherent state, we can express the time-dependence of $S(\theta, \omega, t)$ as

$$
S(\theta, \omega, t)=H\left(\theta, \omega, \alpha(t), \alpha(t)^{*}, \beta(t), \beta(t)^{*}\right)=\sum_{l=-\infty}^{\infty} H_{l}\left(\omega, \alpha(t), \alpha(t)^{*}, \beta(t), \beta(t)^{*}\right) e^{i l \theta}
$$

and restrict (146) - (148) to these solutions by substituting $S=H$. This determines the autonomous four-dimensional system:

$$
\begin{aligned}
& \dot{\alpha}=\lambda \alpha-\pi K\left(\alpha^{*}+\beta+<g, H_{-1}>\right)<\tilde{\psi}, H_{2}> \\
& \dot{\beta}=\lambda \beta-\pi K\left(\alpha+\beta^{*}+<g, H_{1}>\right)<\kappa \cdot \tilde{\psi}, H_{-2}>.
\end{aligned}
$$

For this bifurcation the mode amplitudes transform according to

$$
\begin{aligned}
\phi \cdot(\alpha, \beta) & =\left(e^{-i \phi} \alpha, e^{i \phi} \beta\right) \\
\kappa \cdot(\alpha, \beta) & =(\beta, \alpha),
\end{aligned}
$$

and the $\mathrm{O}(2)$-invariance of the center manifold implies that the Fourier coefficients of $H$ have the form

$$
H_{l}=\sum_{j=0}^{l} \alpha^{j}\left(\beta^{*}\right)^{l-j} H_{l}^{(j)}\left(\omega,|\alpha|^{2},|\beta|^{2}, \alpha \beta, \alpha^{*} \beta^{*}\right)
$$

for $l>0$. Here $H_{l}^{(j)}$ are functions of $|\alpha|^{2},|\beta|^{2}, \alpha \beta, \alpha^{*} \beta^{*}$ and $\omega$ that satisfy

$$
H_{l}^{(j)}\left(-\omega,|\alpha|^{2},|\beta|^{2}, \alpha \beta, \alpha^{*} \beta^{*}\right)^{*}=H_{l}^{(l-j)}\left(\omega,|\beta|^{2},|\alpha|^{2}, \alpha \beta, \alpha^{*} \beta^{*}\right)
$$


for $j=1,2, \ldots, l$. The analysis leading to (154) - (155) is analogous to the argument given above for steady-state bifurcation: the invariance of the manifold requires the Fourier components of $H$ to satisfy

$$
e^{-i l \phi} H_{l}\left(\omega, \alpha, \alpha^{*}, \beta, \beta^{*}\right)=H_{l}\left(\theta, \omega, e^{-i \phi} \alpha, e^{i \phi} \alpha^{*}, e^{i \phi} \beta, e^{-i \phi} \beta^{*}\right),
$$

and

$$
H_{l}\left(-\omega, \alpha, \alpha^{*}, \beta, \beta^{*}\right)^{*}=H_{l}\left(\omega, \beta, \beta^{*}, \alpha, \alpha^{*}\right)
$$

The constraint (156) implies that the function $\left(\alpha^{*}\right)^{l} H_{l}$ is invariant under rotations, hence it can only depend on the mode amplitudes only through the basic rotation invariants $|\alpha|^{2},|\beta|^{2}, \alpha \beta$, and $\alpha^{*} \beta^{*}$ and must contain an overall factor of $\left(\alpha^{*}\right)^{l}$. By combining $|\alpha|^{2}$ and $\alpha^{*} \beta^{*}$ there are $l+1$ distinct ways to generate this factor so $\left(\alpha^{*}\right)^{l} H_{l}$ takes the form

$$
\left(\alpha^{*}\right)^{l} H_{l}=\sum_{j=0}^{l}|\alpha|^{2 j}\left(\alpha^{*} \beta^{*}\right)^{l-j} H_{l}^{(j)}\left(\omega,|\alpha|^{2},|\beta|^{2}, \alpha \beta, \alpha^{*} \beta^{*}\right)
$$

where the functions $H_{l}^{(j)}$ are arbitrary functions of $|\alpha|^{2},|\beta|^{2}, \alpha \beta, \alpha^{*} \beta^{*}$ and $\omega$. Thus $H_{l}$ has the asserted form (154); the condition imposed by reflection invariance (157) implies (155).

For $l=1$ and $l=2$, the components needed in (150) and (151), we have

$$
\begin{gathered}
H_{1}=\alpha H_{1}^{(1)}\left(\omega,|\alpha|^{2},|\beta|^{2}, \alpha \beta, \alpha^{*} \beta^{*}\right)+\beta^{*} H_{1}^{(0)}\left(\omega,|\alpha|^{2},|\beta|^{2}, \alpha \beta, \alpha^{*} \beta^{*}\right) \\
H_{2}=\alpha^{2} H_{2}^{(2)}\left(\omega,|\alpha|^{2},|\beta|^{2}, \alpha \beta, \alpha^{*} \beta^{*}\right)+\alpha \beta^{*} H_{2}^{(1)}\left(\omega,|\alpha|^{2},|\beta|^{2}, \alpha \beta, \alpha^{*} \beta^{*}\right)+ \\
\left(\beta^{*}\right)^{2} H_{2}^{(0)}\left(\omega,|\alpha|^{2},|\beta|^{2}, \alpha \beta, \alpha^{*} \beta^{*}\right) .
\end{gathered}
$$

Since the center manifold geometry requires that the Taylor expansion of $H$ begins at second order, the leading order terms in these components are

$$
\begin{aligned}
& H_{1}=\mathcal{O}(3) \\
& H_{2}=\alpha^{2} h_{2}^{(2)}(\omega)+\alpha \beta^{*} h_{2}^{(1)}(\omega)+\left(\beta^{*}\right)^{2} h_{2}^{(0)}(\omega)+\mathcal{O}(3) ;
\end{aligned}
$$


the third order terms will not be required to determine the cubic terms in the amplitude equations. Note that the general relation (155) implies

$$
\begin{aligned}
& h_{2}^{(2)}(-\omega)^{*}=h_{2}^{(0)}(\omega) \\
& h_{2}^{(1)}(-\omega)^{*}=h_{2}^{(1)}(\omega) .
\end{aligned}
$$

The four-dimensional vector field (150)-(151) commutes with the $\mathrm{O}(2)$ symmetry (152) - (153) on the center manifold; in addition this system can be further simplified by making a nonlinear change of variables $(\alpha, \beta) \rightarrow\left(\alpha^{\prime}, \beta^{\prime}\right)$ to put 150)-(151) in Poincaré-Birkhoff normal form. [17, 18] This change of variables introduces an additional $\mathrm{S}^{1}$ symmetry defined by $(\alpha, \beta) \rightarrow e^{i \chi}(\alpha, \beta)$; this is the "phase shift symmetry" that characterizes the Hopf normal form even in bifurcations without symmetry. 20, 21 Thus the normal form for (151) will have $\mathrm{O}(2) \times \mathrm{S}^{1}$ symmetry and must be of the general form 17, 18

$$
\left(\begin{array}{c}
\dot{\alpha}^{\prime} \\
\dot{\beta}^{\prime}
\end{array}\right)=[p(u, \Delta)+i q(u, \Delta)]\left(\begin{array}{c}
\alpha^{\prime} \\
\beta^{\prime}
\end{array}\right)+[r(u, \Delta)+i s(u, \Delta)] \delta\left(\begin{array}{c}
\alpha^{\prime} \\
-\beta^{\prime}
\end{array}\right),
$$

where $\delta=\left|\beta^{\prime}\right|^{2}-\left|\alpha^{\prime}\right|^{2}$ and $p, q, r$, and $s$ are real-valued functions of the two basic invariants $u=\left|\alpha^{\prime}\right|^{2}+\left|\beta^{\prime}\right|^{2}$ and $\Delta=\delta^{2}$. A final reduction to two dimensions is accomplished by introducing amplitudes and phases $\alpha^{\prime}=x_{1} e^{i \sigma_{1}}$ and $\beta^{\prime}=x_{2} e^{i \sigma_{2}}$ into (165) and noting that the two-dimensional amplitude equations,

$$
\begin{aligned}
& \dot{x}_{1}=[p+\delta r] x_{1} \\
& \dot{x}_{2}=[p-\delta r] x_{2}
\end{aligned}
$$

are decoupled from the phase evolution $\dot{\sigma}_{1}=q+\delta s$ and $\dot{\sigma}_{2}=q-\delta s$. This decoupling is a consequence of the phase shift symmetry. Upon expanding (166) and (167) we obtain the leading order behavior

$$
\begin{aligned}
& \dot{x}_{1}=\left[p(0)+\left(p_{u}(0)-r(0)\right) x_{1}^{2}+\left(p_{u}(0)+r(0)\right) x_{2}^{2}+\cdots\right] x_{1} \\
& \dot{x}_{2}=\left[p(0)+\left(p_{u}(0)+r(0)\right) x_{1}^{2}+\left(p_{u}(0)-r(0)\right) x_{2}^{2}+\cdots\right] x_{2}
\end{aligned}
$$


where $p(0)$ denotes the function $p(u, \Delta)$ evaluated at the origin. From the linear terms in (151) we find $p(0)=\operatorname{Re} \lambda$ and the third order coefficients $p_{u}(0)$ and $r(0)$ are evaluated in the following section.

Assuming the non-degeneracy condition, $r(0) p(0)\left(p_{u}(0)-r(0)\right) \neq 0$, then the amplitude equations (168) - (169) may be truncated at third order. 17] In addition to the incoherent state $\left(x_{1}, x_{2}\right)=(0,0)$, setting $\dot{x}_{1}=\dot{x}_{2}=0$ yields two other types of solutions summarized in Table 1. There are travelling wave (TW) solutions $\left(x_{\mathrm{TW}}, 0\right)$ and $\left(0, x_{\mathrm{TW}}\right)$ with $\operatorname{Re} \lambda+\left(p_{u}(0)-\right.$ $r(0)) x_{\mathrm{TW}}^{2}=0$ and standing wave solutions (SW) $\left(x_{\mathrm{SW}}, x_{\mathrm{SW}}\right)$ with $\operatorname{Re} \lambda+2 p_{u}(0) x_{\mathrm{SW}}^{2}=0$.

In the rotating frame, both $\mathrm{TW}$ solutions have same frequency $\omega_{\mathrm{TW}}=q+x_{\mathrm{TW}}^{2} s$ but the two states propagate oppositely in phase; the $\left(x_{\mathrm{TW}}, 0\right)$ state corresponds to a density depending on $\left(\theta+\omega_{\mathrm{TW}} t\right)$ and the $\left(0, x_{\mathrm{TW}}\right)$ state evolves according to $\left(\theta-\omega_{\mathrm{TW}} t\right)$. Note that $q$ and $s$ are evaluated on the TW solution, and therefore near the bifurcation $\omega_{\mathrm{TW}}$ is fixed by the eigenvalue (91) at criticality $\left(K=K_{h}\right)$ :

$$
\begin{aligned}
\omega_{\mathrm{TW}}^{c} & =q(0)+\text { higher order terms in } x_{\mathrm{TW}}^{2} \\
& \approx \sqrt{\omega_{0}^{2}-(\epsilon+D)^{2}} .
\end{aligned}
$$

In the lab frame (20), the frequencies of these two states are split to $\bar{\omega}-\omega_{\mathrm{TW}}$ and $\bar{\omega}+\omega_{\mathrm{TW}}$, respectively.

The SW frequency is $\omega_{\mathrm{SW}}=q$ where $q$ is evaluated on the SW solution; near criticality $\omega_{\mathrm{SW}}^{c}$ is also given by $(170)$. The physical appearance of the SW can be described from its form near onset,

$$
\begin{aligned}
\rho_{\mathrm{SW}}(\theta, \omega, t)= & \frac{1}{2 \pi}+K x_{\mathrm{SW}} \operatorname{Im}\left[\frac{\alpha_{0}}{\omega+\omega_{\mathrm{SW}}^{c}-i D} e^{i\left(\theta+\omega_{\mathrm{SW}}^{c} t\right)}-\frac{\beta_{0}}{\omega-\omega_{\mathrm{SW}}^{c}+i D} e^{-i\left(\theta-\omega_{\mathrm{SW}}^{c} t\right)}\right] \\
& + \text { higher order terms in } x_{\mathrm{SW}}
\end{aligned}
$$

where $\alpha_{0}$ and $\beta_{0}$ are initial phases. There are two counter-propagating synchronized clumps containing oscillators with native frequencies near $-\omega_{\mathrm{SW}}^{c}$ and $+\omega_{\mathrm{SW}}^{c}$, respectively. Thus 
Table 1. Branching equations and eigenvalues for non-degenerate Hopf bifurcation

$\begin{array}{ccc}\text { Solution type }\left(x_{1}, x_{2}\right) & \underline{\text { Branching Equations }} & \underline{\text { Eigenvalues }} \\ \begin{array}{ccc}\text { Travelling wave }(\mathrm{TW}) \\ \left(x_{\mathrm{TW}}, 0\right) \text { and }\left(0, x_{\mathrm{TW}}\right)\end{array} & \operatorname{Re} \lambda+\left(p_{u}-r\right) x_{\mathrm{TW}}^{2}=0 & 2\left(p_{u}-r\right) x_{\mathrm{TW}}^{2}, \\ \operatorname{Re} \lambda+\left(p_{u}+r\right) x_{\mathrm{TW}}^{2} \\ \begin{array}{c}\text { Standing wave }(\mathrm{SW}) \\ \left(x_{\mathrm{SW}}, x_{\mathrm{SW}}\right)\end{array} & \operatorname{Re} \lambda+2 p_{u} x_{\mathrm{SW}}^{2}=0 & 4 p_{u} x_{\mathrm{SW}}^{2}, \\ & & -4 r x_{\mathrm{SW}}^{2}\end{array}$

\section{NOTES:}

The coefficients $p_{u}$ and $r$ are evaluated at $\left(x_{1}, x_{2}\right)=(0,0)$.

the population exhibits two simultaneous macroscopic oscillations. In the lab frame these macroscopic oscillators appear as a slow oscillator at frequency $\bar{\omega}-\omega_{\mathrm{SW}}$ and a fast oscillator at $\bar{\omega}+\omega_{\mathrm{SW}}$.

The direction of bifurcation for these waves is determined by $\left(p_{u}(0)-r(0)\right)$ and $p_{u}(0)$. Their linear stabilities are easily calculated: the TW eigenvalues are $2\left(p_{u}(0)-r(0)\right) x_{\mathrm{TW}}^{2}$ and $\operatorname{Re} \lambda+\left(p_{u}(0)+r(0)\right) x_{\mathrm{TW}}^{2}$; the SW eigenvalues are $4 p_{u}(0) x_{\mathrm{SW}}^{2}$ and $-4 r(0) x_{\mathrm{SW}}^{2}$. Depending on the signs and relative magnitudes of $p_{u}(0)$ and $r(0)$ there are six possible bifurcations as shown in Fig. 2. For the bimodal population (85) we shall see that $\left(p_{u}(0)-r(0)\right)$ and $p_{u}(0)$ are negative while $r(0)$ is positive. Thus the SW are supercritical and stable and the TW are supercritical and unstable.

\subsubsection{Evaluation of $p_{u}$ and $r$}

From (162) for $H_{2}$,

$$
<\tilde{\psi}, H_{2}>=\alpha^{2}<\tilde{\psi}, h_{2}^{(2)}>+\alpha \beta^{*}<\tilde{\psi}, h_{2}^{(1)}>+\left(\beta^{*}\right)^{2}<\tilde{\psi}, h_{2}^{(0)}>+\mathcal{O}(3)(172)
$$




$$
\begin{gathered}
<\kappa \cdot \tilde{\psi}, H_{-2}>=\left(\alpha^{*}\right)^{2}<\kappa \cdot \tilde{\psi}, h_{2}^{(2) *}>+\alpha^{*} \beta<\kappa \cdot \tilde{\psi}, h_{2}^{(1) *}>+ \\
\beta^{2}<\kappa \cdot \tilde{\psi}, h_{2}^{(0) *}>+\mathcal{O}(3)
\end{gathered}
$$

which determines the nonlinear terms in (150)-(151) through third order:

$$
\begin{array}{r}
\left(\begin{array}{c}
\dot{\alpha} \\
\dot{\beta}
\end{array}\right)= \\
\lambda\left(\begin{array}{c}
\alpha \\
\beta
\end{array}\right)-\pi K\left(\begin{array}{c}
\alpha\left[<\tilde{\psi}, h_{2}^{(2)}>|\alpha|^{2}+<\tilde{\psi}, h_{2}^{(1)}>|\beta|^{2}\right] \\
\beta\left[<\kappa \tilde{\psi}, h_{2}^{(0) *}>|\beta|^{2}+<\kappa \cdot \tilde{\psi}, h_{2}^{(1) *}>|\alpha|^{2}\right]
\end{array}\right) \\
\quad-\pi K\left(\begin{array}{c}
\alpha^{*}\left[\alpha \beta^{*}<\tilde{\psi}, h_{2}^{(1)}>+\left(\beta^{*}\right)^{2}<\tilde{\psi}, h_{2}^{(0)}>\right] \\
\beta^{*}\left[\left(\alpha^{*}\right)^{2}<\kappa \cdot \tilde{\psi}, h_{2}^{(2) *}>+\alpha^{*} \beta<\kappa \cdot \tilde{\psi}, h_{2}^{(1) *}>\right]
\end{array}\right) \\
-\pi K\left(\begin{array}{c}
\beta\left[\alpha^{2}<\tilde{\psi}, h_{2}^{(2)}>+\left(\beta^{*}\right)^{2}<\tilde{\psi}, h_{2}^{(0)}>\right] \\
\alpha\left[\left(\alpha^{*}\right)^{2}<\kappa \cdot \tilde{\psi}, h_{2}^{(2) *}>+\beta^{2}<\kappa \cdot \tilde{\psi}, h_{2}^{(0) *}>\right]
\end{array}\right)+\mathcal{O}(4)
\end{array}
$$

Only the first set of nonlinear terms in (174) have the phase shift symmetry and are retained in the normal form:

$$
\begin{gathered}
\left(\begin{array}{c}
\dot{\alpha}^{\prime} \\
\dot{\beta}^{\prime}
\end{array}\right)=\lambda\left(\begin{array}{c}
\alpha^{\prime} \\
\beta^{\prime}
\end{array}\right)-\pi \\
+\left(\begin{array}{c}
\alpha^{\prime}\left[<\tilde{\psi}, h_{2}^{(2)}>\left|\alpha^{\prime}\right|^{2}+<\tilde{\psi}, h_{2}^{(1)}>\left|\beta^{\prime}\right|^{2}\right] \\
\beta^{\prime}\left[<\kappa \cdot \tilde{\psi}, h_{2}^{(0) *}>\left|\beta^{\prime}\right|^{2}+<\kappa \cdot \tilde{\psi}, h_{2}^{(1) *}>\left|\alpha^{\prime}\right|^{2}\right]
\end{array}\right) \\
+\mathcal{O}(5) ;
\end{gathered}
$$

the remaining cubic terms and all fourth order terms in (174) are removed by the normal form change of variables $(\alpha, \beta) \rightarrow\left(\alpha^{\prime}, \beta^{\prime}\right)$. From (163) and (164) the coefficients in (175) are related: $\left\langle\tilde{\psi}, h_{2}^{(2)}>=<\kappa \cdot \tilde{\psi}, h_{2}^{(0) *}>\right.$ and $<\tilde{\psi}, h_{2}^{(1)}>=<\kappa \cdot \tilde{\psi}, h_{2}^{(1) *}>$ so that the result in (175) can be rewritten in the standard form (165). Noting that $\left|\alpha^{\prime}\right|^{2}=(u-\delta) / 2$ and $\left|\beta^{\prime}\right|^{2}=(u+\delta) / 2$, (175) becomes

$$
\begin{aligned}
\left(\begin{array}{c}
\dot{\alpha}^{\prime} \\
\dot{\beta}^{\prime}
\end{array}\right)= & {\left[\lambda-\frac{\pi K}{2}\left(<\tilde{\psi}, h_{2}^{(2)}>+<\tilde{\psi}, h_{2}^{(1)}>\right) u+\mathcal{O}(4)\right]\left(\begin{array}{c}
\alpha^{\prime} \\
\beta^{\prime}
\end{array}\right) } \\
& +\left[\frac{\pi K}{2}\left(<\tilde{\psi}, h_{2}^{(2)}>-<\tilde{\psi}, h_{2}^{(1)}>\right)+\mathcal{O}(2)\right] \delta\left(\begin{array}{c}
\alpha^{\prime} \\
-\beta^{\prime}
\end{array}\right) .
\end{aligned}
$$


By comparing (165) and (176) we can identify the cubic coefficients $p_{u}$ and $r$

$$
\begin{aligned}
p_{u}(0) & =-\frac{\pi K}{2} \operatorname{Re}<\tilde{\psi},\left[h_{2}^{(1)}+h_{2}^{(2)}\right]> \\
r(0) & =-\frac{\pi K}{2} \operatorname{Re}<\tilde{\psi},\left[h_{2}^{(1)}-h_{2}^{(2)}\right]>
\end{aligned}
$$

in terms of the center manifold coefficients $h_{2}^{(2)}$ and $h_{2}^{(1)}$.

For this bifurcation the equation for $H$ (127) becomes

$$
\begin{aligned}
& {\left[\frac{\partial H}{\partial \alpha} \dot{\alpha}+\frac{\partial H}{\partial \beta} \dot{\beta}+c c\right]=} \\
& \quad \mathcal{L} H-\pi K\left\{e^{i \theta}\left(\alpha^{*}+\beta+<g, H_{-1}>\right)\left[H_{2}-<\tilde{\psi}, H_{2}>\psi-<\kappa \cdot \tilde{\psi}^{*}, H_{2}>\kappa \cdot \psi^{*}\right]\right. \\
& \quad+2 e^{i 2 \theta}\left[\left(\alpha^{*}+\beta+<g, H_{-1}>\right) H_{3}(\omega)-\left(\alpha+\beta^{*}+<g, H_{1}>\right)\left(\alpha \psi+\beta^{*} \kappa \cdot \psi^{*}+H_{1}\right)\right] \\
& \left.\quad+\sum_{l \geq 3} l e^{i l \theta}\left[\left(\alpha^{*}+\beta+<g, H_{-1}>\right) H_{l+1}(\omega)-\left(\alpha+\beta^{*}+<g, H_{1}>\right) H_{l-1}(\omega)\right]+c c\right\}
\end{aligned}
$$

and the functions $h_{2}^{(2)}, h_{2}^{(1)}$, and $h_{2}^{(0)}$ may be determined from (179). By inspection of the $l=2$ component we note that the inhomogeneous terms $2 \pi K e^{i 2 \theta}\left(\alpha+\beta^{*}\right)\left(\alpha \psi+\beta^{*} \kappa \cdot \psi^{*}\right)$ in (179) generate the leading terms $h_{2}^{(i)}(\omega)$ in $H_{2}$; since $H_{l}(\omega) \sim \mathcal{O}(3)$ for $l \neq 2$, applying (162) to the $l=2$ component of (179) and equating terms of the same order, we obtain

$$
\begin{aligned}
2 \lambda h_{2}^{(2)} & =\mathrm{L}_{2} h_{2}^{(2)}+2 \pi K \psi \\
\left(\lambda+\lambda^{*}\right) h_{2}^{(1)} & =\mathrm{L}_{2} h_{2}^{(1)}+2 \pi K\left(\psi+\kappa \cdot \psi^{*}\right) \\
2 \lambda^{*} h_{2}^{(0)} & =\mathrm{L}_{2} h_{2}^{(0)}+2 \pi K \kappa \cdot \psi^{*},
\end{aligned}
$$

with solutions

$$
\begin{aligned}
h_{2}^{(2)}(\omega) & =\frac{-i \pi K \psi(\omega)}{\omega-z_{0}-i 2 D} \\
h_{2}^{(1)}(\omega) & =\frac{-i \pi K\left(\psi(\omega)+\kappa \cdot \psi(\omega)^{*}\right)}{\omega-i \operatorname{Im} z_{0}-i 2 D} \\
h_{2}^{(0)}(\omega) & =\frac{-i \pi K \kappa \cdot \psi(\omega)^{*}}{\omega+z_{0}^{*}-i 2 D} .
\end{aligned}
$$


These results satisfy the relations (163) - (164).

From (177) - (178) these results determine the cubic coefficients explicitly

$$
\begin{aligned}
p_{u}(0) & =\frac{-\pi^{2} K^{2}}{2} \operatorname{Im} \int_{-\infty}^{\infty} d \omega \tilde{\psi}(\omega)^{*}\left[\frac{\psi(\omega)+\kappa \cdot \psi(\omega)^{*}}{\left(\omega-i \operatorname{Im} z_{0}-i 2 D\right)}+\frac{\psi(\omega)}{\left(\omega-z_{0}-i 2 D\right)}\right] \\
r(0) & =\frac{-\pi^{2} K^{2}}{2} \operatorname{Im} \int_{-\infty}^{\infty} d \omega \tilde{\psi}(\omega)^{*}\left[\frac{\psi(\omega)+\kappa \cdot \psi(\omega)^{*}}{\left(\omega-i \operatorname{Im} z_{0}-i 2 D\right)}-\frac{\psi(\omega)}{\left(\omega-z_{0}-i 2 D\right)}\right] .
\end{aligned}
$$

Provided $\operatorname{Im} z_{0}>-D$, any singularities in the lower half plane are due to $g(\omega)$. Hence by inspection we can see that at criticality for the bifurcation $\left(\operatorname{Im} z_{0}=0\right)$, the limit $D \rightarrow$ 0 is finite for both $p_{u}(0)$ and $r(0)$. By contrast the corresponding limit for the Vlasov equation involves pinching singularities that produce a singular $D^{-3}$ behavior. [16] Here the well-behaved limit at $D=0$ suggests that the oscillations produced by the bifurcation will grow like $\sqrt{\left|K-K_{c}\right|}$ even in the absence of noise.

\subsubsection{Example of a bimodal population}

We illustrate these results for the bimodal population described in (85); for $4 \omega_{0}>K$ the

eigenvalues $\left(\lambda, \lambda^{*}\right)$ are given by $\lambda=-i z_{0}=\left(K-K_{h}\right) / 4 \pm i \Omega$ where $\Omega \equiv \sqrt{\omega_{0}^{2}-(K / 4)^{2}}$ and $K_{h} \equiv 4(\epsilon+D)$. If $K$ increases above $K_{h}$, there is a Hopf bifurcation. The coefficients $p_{u}(0)$ and $r(0)$ can be evaluated analytically at the bifurcation $K=K_{h}$; by closing the integration contours in the lower half plane and using $\Omega^{2}=\omega_{0}^{2}-(\epsilon+D)^{2}$ one obtains

$$
\begin{aligned}
& \Lambda_{1}^{\prime}\left(z_{0}\right)=\frac{i K_{h}}{2} \frac{\omega_{0}^{2}+(\Omega-i(\epsilon+D))^{2}}{\left[\omega_{0}^{2}-(\Omega-i(\epsilon+D))^{2}\right]^{2}} \\
& \operatorname{Re}<\tilde{\psi}, h_{2}^{(1)}>=\frac{16 \pi K_{h} D(\epsilon+D)^{2} \Omega^{2} \omega_{0}^{4}}{\left|\omega_{0}+i(\epsilon+2 D)\right|^{2}\left|\omega_{0}^{4}-(\Omega+i(\epsilon+D))^{4}\right|^{2}} \\
& \operatorname{Re}<\tilde{\psi}, h_{2}^{(2)}>=\frac{\left.\left.4 \pi K_{h} \Omega^{2}(\epsilon+2 D) \omega_{0}^{2}\left[4 \omega_{0}^{2}+(\epsilon+2 D)\right)^{2}-(\epsilon+D)\right)^{2}\right]}{\left|\omega_{0}^{2}+(\Omega-i(\epsilon+D))^{2}\right|^{2}\left|\omega_{0}^{2}-(\Omega-i(\epsilon+2 D))^{2}\right|^{2}} .
\end{aligned}
$$


The direction of bifurcation and stability of the travelling waves and standing waves are controlled by $p_{u}(0), r(0)$, and $p_{u}(0)-r(0)$. Since $\operatorname{Re}<\tilde{\psi}, h_{2}^{(1)}>$ and $\operatorname{Re}<\tilde{\psi}, h_{2}^{(2)}>$ are clearly positive, one can see by inspection that both $p_{u}(0)$ and $p_{u}(0)-r(0)$ are negative, and the SW and TW solutions are always supercritical. The bifurcation produces stable TW solutions if $r(0)<0$ and stable SW if $r(0)>0$; see Fig. 2. Subtracting $\operatorname{Re}<\tilde{\psi}, h_{2}^{(2)}>$ from $\operatorname{Re}<\tilde{\psi}, h_{2}^{(1)}>$ gives

$$
r(0)=\frac{8 \pi^{2} K_{h}^{2} \Omega^{2} \omega_{0}^{4}(\epsilon+D)^{2} P\left(\epsilon, D, \omega_{0}^{2}\right)}{\left|\omega_{0}^{4}-(\Omega+i(\epsilon+D))^{4}\right|^{2}\left|\omega_{0}^{2}-(\Omega-i(\epsilon+2 D))^{2}\right|^{2}\left|\omega_{0}+i(\epsilon+2 D)\right|^{2}}
$$

where

$$
\begin{aligned}
P\left(\epsilon, D, \omega_{0}^{2}\right)= & 4(\epsilon+2 D) \omega_{0}^{4}+(\epsilon+2 D)\left[4 \epsilon^{2}+14 \epsilon D+11 D^{2}\right] \omega_{0}^{2} \\
& +D(\epsilon+D)(3 \epsilon+3 D)\left[\epsilon^{2}+5 \epsilon D+5 D^{2}\right] .
\end{aligned}
$$

Thus for this bimodal population $r(0)$ is always positive and the bifurcation always leads to stable standing waves.

In light of Kuramoto's earlier discussion of the partially synchronized bimodal population (cf. the remarks in Section 2.3), it is interesting to compare the frequencies $\omega_{\text {peak }}$ in (86) and $\omega_{\mathrm{SW}}^{c}$ in $(170)$ for the SW near onset in the relevant frequency range $(1+D / \epsilon)<\omega_{0} / \epsilon<\infty$. From (86) and (170) we find

$$
\left(\frac{\omega_{\mathrm{SW}}^{c}}{\omega_{\text {peak }}}\right)^{2}=\frac{\left(\omega_{0} / \epsilon\right)^{2}-(1+D / \epsilon)^{2}}{\left[\left(2 \omega_{0} / \epsilon\right)-\sqrt{1+\left(\omega_{0} / \epsilon\right)^{2}}\right] \sqrt{1+\left(\omega_{0} / \epsilon\right)^{2}}} ;
$$

as the peaks move far apart $\left(\omega_{0} / \epsilon \rightarrow \infty\right)$ this ratio approaches unity and the oscillators in the peaks are indeed the oscillators that synchronize. However as $\omega_{0} / \epsilon$ approaches $(1+D / \epsilon)$ the oscillators comprising the standing wave synchronize at frequencies that are substantially shifted away from the peaks of the native distribution towards the interior of the distribution; this is illustrated in Fig. 3. 


\subsubsection{Comparison to Bonilla, Neu, and Spigler}

Bonilla, et al. have previously calculated the amplitude equation for a Hopf bifurcation from the incoherent state, but they neglected to include the second eigenvector $\kappa \cdot \Psi$ in (57) that is forced by $\mathrm{O}(2)$ symmetry. In our notation this omission has the effect of setting the amplitude $\beta(t)$ of this mode to zero; if this is done then the two-dimensional system for $\left(x_{1}, x_{2}\right)$ in $(168)-(169)$ is reduced to

$$
\dot{x}_{1}=\left[p(0)+\left(p_{u}(0)-r(0)\right) x_{1}^{2}+\cdots\right] x_{1} .
$$

Since $p_{u}(0)-r(0)<0$ for the bimodal population, this equation predicts a single TW $\left(x_{1}, x_{2}\right)=\left(x_{\mathrm{TW}}, 0\right)$ which is supercritical and stable. The SW solutions $\left(x_{1}, x_{2}\right)=\left(x_{\mathrm{SW}}, x_{\mathrm{SW}}\right)$ and the second TW solution $\left(x_{1}, x_{2}\right)=\left(0, x_{\mathrm{TW}}\right)$ are omitted; in addition the actual instability of the TW is missed because the TW eigenvalue $\operatorname{Re} \lambda+\left(p_{u}(0)+r(0)\right) x_{\mathrm{TW}}^{2}$ corresponding to perturbations in the direction of the SW state is not included.

\subsubsection{Comparison to Okuda and Kuramoto}

Okuda and Kuramoto consider two populations of oscillators $(i=1,2)$ with number densities $n_{i}(\phi, t)$; in the absence of couplings each population has a definite native frequency: $\omega_{1}=\bar{\omega}+\Delta \omega / 2$ and $\omega_{2}=\bar{\omega}-\Delta \omega / 2$ respectively. The evolution of $n_{1}(\phi, t)$ and $n_{2}(\phi, t)$ is described by

$$
\begin{aligned}
\frac{\partial n_{1}}{\partial t}= & -\omega_{1} \frac{\partial n_{1}}{\partial \phi}+\frac{\partial}{\partial \phi}\left\{n_{1}(\phi, t) \int_{0}^{2 \pi} d \phi^{\prime} \sin \left(\phi-\phi^{\prime}\right)\left[K_{1}^{\prime} n_{1}\left(\phi^{\prime}, t\right)+K^{\prime} n_{2}\left(\phi^{\prime}, t\right)\right]\right\} \\
& +D \frac{\partial^{2} n_{1}}{\partial \phi^{2}} \\
\frac{\partial n_{2}}{\partial t}= & -\omega_{2} \frac{\partial n_{2}}{\partial \phi}+\frac{\partial}{\partial \phi}\left\{n_{2}(\phi, t) \int_{0}^{2 \pi} d \phi^{\prime} \sin \left(\phi-\phi^{\prime}\right)\left[K_{2}^{\prime} n_{2}\left(\phi^{\prime}, t\right)+K^{\prime} n_{1}\left(\phi^{\prime}, t\right)\right]\right\} \\
& +D \frac{\partial^{2} n_{2}}{\partial \phi^{2}}
\end{aligned}
$$

\footnotetext{
${ }^{5}$ This corresponds to equation (3.18a) in Bonilla, et al. with the identification $K x_{1} / 2 \rightarrow|A|$.
} 
The intra-population couplings are $K_{1}^{\prime}$ and $K_{2}^{\prime}$ and the coupling between the populations is $K^{\prime}$. The incoherent state is an equilibrium described by $n_{1}(\phi)=n_{2}(\phi)=1 / 2 \pi$, and the authors analyzed bifurcations from this state. This model has $O(2)$ symmetry if $\bar{\omega}=0$ and $K_{1}^{\prime}=K_{2}^{\prime}$.

For the bimodal population (85) at $\epsilon=0$, the Fokker-Planck equation (4) reduces to the O-K model but with the constraint of equal couplings $K_{1}^{\prime}=K_{2}^{\prime}=K^{\prime}$. For the $\epsilon=0$ distribution,

$$
g(\omega)=\frac{1}{2}\left[\delta\left(\omega+\omega_{0}\right)+\delta\left(\omega-\omega_{0}\right)\right],
$$

we define number densities by integrating over the frequency distribution of each subpopulation

$$
\begin{aligned}
& n_{1}(\theta, t)=\int_{-\infty}^{\infty} d \omega \delta\left(\omega-\omega_{0}\right) \rho(\theta, \omega, t) \\
& n_{2}(\theta, t)=\int_{-\infty}^{\infty} d \omega \delta\left(\omega+\omega_{0}\right) \rho(\theta, \omega, t)
\end{aligned}
$$

so that (田) becomes

$$
\begin{aligned}
\frac{\partial n_{1}}{\partial t}= & -\omega_{0} \frac{\partial n_{1}}{\partial \theta}+\frac{\partial}{\partial \theta}\left\{n_{1}(\theta, t) \int_{0}^{2 \pi} d \theta^{\prime} \sin \left(\theta-\theta^{\prime}\right)\left[(K / 2) n_{1}\left(\theta^{\prime}, t\right)+(K / 2) n_{2}\left(\theta^{\prime}, t\right)\right]\right\} \\
& +D \frac{\partial^{2} n_{1}}{\partial \theta^{2}} \\
\frac{\partial n_{2}}{\partial t}= & +\omega_{0} \frac{\partial n_{2}}{\partial \theta}+\frac{\partial}{\partial \theta}\left\{n_{2}(\theta, t) \int_{0}^{2 \pi} d \theta^{\prime} \sin \left(\theta-\theta^{\prime}\right)\left[(K / 2) n_{2}\left(\theta^{\prime}, t\right)+(K / 2) n_{1}\left(\theta^{\prime}, t\right)\right]\right\} \\
& +D \frac{\partial^{2} n_{2}}{\partial \theta^{2}} .
\end{aligned}
$$

The equations (195) - (196) have this form if we set $\Delta \omega=2 \omega_{0}$ and $K_{1}^{\prime}=K_{2}^{\prime}=K^{\prime}=K / 2$ and transform to the rotating frame $\theta=\phi-\bar{\omega} t$ where average frequency is zero. One of the cases considered by Okuda and Kuramoto is $K_{1}^{\prime}=K_{2}^{\prime}=1$ with $K^{\prime}$ and $\Delta \omega$ variable. Their results for the steady state bifurcation and Hopf bifurcation are consistent with those of this 
paper. In particular, for the Hopf bifurcation they find that the SW solutions are expected to be stable.

\subsubsection{Perturbing $\mathrm{O}(2) \rightarrow \mathrm{SO}(2)$}

If the distribution $g(\omega)$ is perturbed so that the reflection symmetry (18) is broken, then the complex eigenvalues of multiplicity-two split into two non-degenerate complex conjugate pairs. The Hopf bifurcation considered here perturbs to two distinct Hopf bifurcations corresponding to the distinct pairs of eigenvalues. The resulting Hopf bifurcations individually lead to time-periodic states in the form of rotating or travelling waves and the interaction between these waves yields modulated waves via secondary bifurcations. 224]-27] The modulated waves are the perturbed form of the standing waves discussed above; Knobloch discusses such perturbed bifurcations in more detail. [14]

\section{Discussion}

The bifurcation analysis is summarized in Fig. 4 which shows the stable nonlinear states that arise from instabilities of the incoherent state. Our discussion does not treat the transition from the stable partially synchronized state to the stable standing waves; this transition is represented by a dashed boundary in Fig. 4. The interaction of the standing waves and the synchronized state can be studied by analyzing the codimension-two bifurcation defined by the merger of the Hopf and steady state bifurcations. The linear theory of the nonsemisimple real eigenvalue was developed in Section 2 of this paper and a normal form theory for the $\mathrm{O}(2)$ Takens-Bogdanov bifurcation has been given by Dangelmayr and Knobloch 28]. One complicating feature of the bimodal population is the subcriticality of the steady state bifurcation at the codimension-two point; one would like to include the stable partially synchronized state at finite amplitude in the analysis. This could be done by generalizing the 
Dangelmayr-Knobloch theory to combine the nonlinear degeneracy of the steady state bifurcation with the linear degeneracy. Formally this leads to a codimension-three bifurcation and would require a normal form of higher order; the analysis of Dangelmayr and Knobloch truncates the normal form equations after cubic terms.

\section{$5 \quad$ Acknowledgements}

I have enjoyed helpful conversations with Steve Strogatz.

\section{$6 \quad$ Figure captions}

1. Eigenvalues of the incoherent state as a function of $\epsilon, D, K$, and $\omega_{0}$. The solid curves indicate parameter values at which there is an eigenvalue on the imaginary axis; along $K=K_{c}$ there is an eigenvalue at zero and along $K=K_{h}$ there is a pure imaginary complex-conjugate pair. At the juncture $K=4(\epsilon+D)$ and $\omega_{0}=(\epsilon+D)$, is the codimension two point where the Hopf bifurcation surface intersects the steady state bifurcation surface. The dashed curves indicate parameter values where eigenvalues coalesce with the continuum; upon crossing $K=K_{e}^{\prime}$ or $K=K_{e}$ an eigenvalue either appears or disappears. Along the dot-dashed line $K=4 \omega_{0}$, the two eigenvalues collide on the real axis. The inset diagrams show the qualitative features of the eigenvalue spectrum in each region. The continuous spectrum at $-D$ is not drawn.

2. Bifurcation diagrams for Hopf bifurcation with $\mathrm{O}(2)$ symmetry. The stability and direction of bifurcation for the travelling waves (TW) and standing waves (SW) are determined by the two cubic coefficients $r(0)$ and $p_{u}(0)$. The incoherent state is the horizontal branch and the two TW solutions are drawn as a single branch. Stable solutions are solid branches and unstable solutions are dashed. The signs of the real 
parts of the eigenvalues are indicated; see Table 1 for the TW and SW eigenvalues.

3. Comparison of the standing wave frequency $\omega_{\mathrm{SW}}^{c}$ and $\omega_{\text {peak }}$ for the bimodal distribution at criticality $K=K_{h}$ for the Hopf bifurcation along the line $(1+D / \epsilon)<\left(\omega_{0} / \epsilon\right)<\infty$. Also shown is the ratio of $\omega_{0}$ to $\omega_{\text {peak }}$; the curves are drawn for $D / \epsilon=1$.

4. Nonlinear states for the bimodal distribution: the incoherent state (I), the partially synchronized state (PS), and the standing waves (SW). The solid lines are the stability boundaries for steady state bifurcation and Hopf bifurcation. The boundary between the standing waves and the partially synchronized state is shown schematically as a dashed line; the precise nature and location of this boundary has not been determined. 


\section{References}

[1] A. Winfree, Biological rhythms and the behavior of populations of coupled oscillators, J. Theor. Biol. 16 (1967) 15-42.

[2] Y. Kuramoto, Chemical Oscillations, Waves, and Turbulence, Springer-Verlag, New York (1984).

[3] H. Sakaguchi, Cooperative phenomena in coupled oscillator systems under external fields, Prog. Theor. Phys. 79 (1988) 39-46.

[4] Y. Kuramoto, Cooperative dynamics of oscillator community, Prog. Theo. Phys. Supp. 79 (1984) 223-240.

[5] Y. Kuramoto and I. Nishikawa, Statistical macrodynamics of large dynamical systems. Case of a phase transition in oscillator communities, J. Stat. Phy. 49 (1987) 569-605.

[6] H. Daido, Intrinsic fluctuations and a phase transition in a class of large populations of interacting oscillators, J. of Stat. Phys. 60 (1990) 753-800.

[7] S. Strogatz and R. Mirollo, Stability of incoherence in a population of coupled oscillators, J. of Stat. Phys. 63 (1991) 613-635.

[8] S. Strogatz, R. Mirollo and P.C. Matthews, Coupled nonlinear oscillators below the synchronization threshold: relaxation by generalized Landau damping, Phys. Rev. Lett. 68 (1992) 2730-2733.

[9] K. Okuda and Y. Kuramoto, Mutual entrainment between populations of coupled oscillators, Prog. Theor. Phys. 86 (1991) 1159-1176. 
[10] H. Daido, Order function and macroscopic mutual entrainment in uniformly coupled limit-cycle oscillators, Prog. Theor. Phys. 88 (1992) 1213-1218.

[11] L.L. Bonilla, J.C. Neu, and R. Spigler, Nonlinear stability of incoherence and collective synchronization in a population of coupled oscillators, J. Stat. Phys. 67 (1992) 313-330.

[12] G.B. Ermentrout, Synchronization in a pool of mutually coupled oscillators with random frequencies, J. Math. Biology 22 (1985) 1-9.

[13] J.L. van Hemmen and W.F. Wreszinski, Lyapunov function for the Kuramoto model of nonlinearly coupled oscillators, J. Stat. Phy. 72 (1993) 145-166.

[14] An interesting discussion of the consequences of reducing $\mathrm{O}(2)$ symmetry to $\mathrm{SO}(2)$ has been given in the context of fluid dynamics by E. Knobloch, Bifurcations in rotating systems, in Theory of Solar and Planetary Dynamos: Introductory Lectures, M.R.E. Proctor and A.D. Gilbert, eds., Cambridge University Press, 1992, in press.

[15] J.D. Crawford and P.D. Hislop, Application of the method of spectral deformation to the Vlasov-Poisson system, Ann. Phys. 189 (1989) 265-317.

[16] J.D. Crawford, Amplitude equations on unstable manifolds: singular behavior from neutral modes, in Modern Mathematical Methods in Transport Theory (Operator Theory: Advances and Applications, vol. 51), W. Greenberg and J. Polewczak, eds., Birkhauser Verlag, Basel, 1991, pp. 97-108.

[17] M. Golubitsky, I. Stewart, and D.G. Schaeffer, Singularities and Groups in Bifurcation Theory: Vol. II, Appl. Math. Sci. 69, Springer-Verlag, New York (1988).

[18] J.D. Crawford and E. Knobloch, Symmetry and symmetry-breaking bifurcations in fluid dynamics, Annu. Rev. Fluid Mech. 23 (1991) 341-387. 
[19] M. Reed and B. Simon, Methods of Modern Mathematical Physics: Vol. IV, Academic Press, New York (1978).

[20] J.D. Crawford, Introduction to bifurcation theory, Rev. Mod. Phys. 63 (1991) 991-1037.

[21] J. Guckenheimer and P. Holmes, 1986. Nonlinear Oscillations, Dynamical Systems, and Bifurcations of Vector Fields (Second Printing). New York: SpringerVerlag. 453 pp.

[22] A. Vanderbauwhede and G. Iooss, 1992, Centre manifold theory in infinite dimensions, Dynamics Reported, Vol. 1, Springer-Verlag, New York, 125-163.

[23] D. Ruelle, Bifurcations in the presence of a symmetry group, Arch. Rational Mech. Anal. 51 (1973) 136.

[24] A.K. Bajaj, Bifurcating periodic solutions in rotationally symmetric systems, SIAM J. Appl. Math. 42 (1982) 1978-1090.

[25] S.A. van Gils and J. Mallet-Paret, Hopf bifurcation and symmetry: travelling and standing waves on the circle, Proc. R. Soc. Edinburgh 104A (1986) 279-307.

[26] W. Nagata, Symmetric Hopf bifurcation and magnetoconvection, Contemporary Math. 56 (1986) 237-265.

[27] P. Peplowski and H. Haken, Effects of detuning on Hopf bifurcation at double eigenvalues in laser systems, Phys. Lett A 120 (1987) 138-140.

[28] G. Dangelmayr and E. Knobloch, The Takens-Bogdanov bifurcation with O(2) symmetry Phil. Trans. Roy. Soc. London A 322 (1987) 243-279. 